\title{
Detection and 3D Visualization of Brain Tumor using Deep Learning and Polynomial Interpolation
}

\author{
by \\ Md. Akram Hossan Tuhin \\ 14301079 \\ Tarunya Pramanick \\ 15101087 \\ Humayoun Kabir Emon \\ 18241051 \\ Wasiur Rahman \\ 14301102
}

A thesis submitted to the Department of Computer Science and Engineering in partial fulfillment of the requirements for the degree of B.Sc. in Computer Science
Department of Computer Science and Engineering Brac University
April 2019

(c) 2019. Brac University

All rights reserved. 


\section{Declaration}

It is hereby declared that

1. The thesis submitted is my/our own original work while completing degree at Brac University.

2. The thesis does not contain material previously published or written by a third party, except where this is appropriately cited through full and accurate referencing.

3. The thesis does not contain material which has been accepted, or submitted, for any other degree or diploma at a university or other institution.

4. We have acknowledged all main sources of help.

\section{Student's Full Name \& Signature:}

Md. Akram Hossan Tuhin 14301079

Humayoun Kabir Emon 18241051
Tarunya Pramanick 15101087

Wasiur Rahman

14301102 


\title{
Approval
}

The thesis/project titled "Detection and 3D Visualization of Brain Tumor using Deep Learning and Polynomial Interpolation" submitted by

1. Md. Akram Hossan Tuhin (14301079)

2. Tarunya Pramanick (15101087)

3. Humayoun Kabir Emon (18241051)

4. Wasiur Rahman (14301102)

Of Spring, 2019 has been accepted as satisfactory in partial fulfillment of the requirement for the degree of B.Sc. in Computer Science on August 23, 2015.

\section{Examining Committee:}

Supervisor:

(Member)

\author{
Dr. Md. Ashraful Alam \\ Assistant Professor \\ Department of Computer Science and Engineering \\ Brac University
}

Program Coordinator:

(Member)

Dr. Jia Uddin

Assistant Professor

Department of Computer Science and Engineering

Brac University

Head of Department:

(Chair)

\author{
Md. Abdul Mottalib \\ Chairperson \\ Department of Computer Science and Engineering \\ Brac University
}




\section{Ethics Statement (Optional)}

This is optional, if you don't have an ethics statement then omit this page 


\section{Abstract}

Among different imaging techniques MRI, MRSI and CT scans are some of the widely use techniques to visualize brain structures to point out brain anomalies especially brain tumor. Identification of brain tumor accurately in clinical practices has always been a hard decision for neurologist as multiple exceptions might present in images which may lead dubious suggestion from neurologist.In our proposed model we are aiming towards brain tumor detection and $3 \mathrm{~d}$ visualization of tumor more accurately in efficient way. Our proposed model composed of three stages such as classification of image using CNN whether any tumor exists of not; segmentation using multi thresholding to extract the detected tumor; and 3d visualization using polynomial interpolation. the proposed model enables enhancing the accuracy of tumor detection as compare to existing models as well as segmenting and 3d visualizing the detected tumor. we get $85 \%$ accuracy on our model comparing with others which is slightly more efficient in terms of classification and detection.

Keywords: CNN, Segmentation, 3D Visualization, Brain Tumor,Polynomial Interpolation, Otsu's Multithresholding. 


\section{Dedication}

We would like to dedicate our work to our parents and supervisor without whom this thesis would have been completed much earlier. 


\section{Acknowledgement}

In the beginning, we would like to thank the almighty Allah for enabling us to conduct our research and allowing us to successfully conclude it.Moreover, we would like to express our gratitude and appreciation to all those outstanding person who collaborated us throughout this research period. First of all, special thanks are due to Assistant Professor Dr. Md. Ashraful Alam, our thesis supervisor. He gave us invaluable advice, provided insight and helped us to implement this research work by providing helpful instructions about how to proceed with our work. In times of severe difficulties, he inspired us well and helped us to overcome the situation.Last but not least, we are thankful to the faculties, seniors, friends and our beloved family who have motivated and inspired us throughout this journey. We should also appreciate all sorts of knowledge we could acquire from various resources from the internet from the work of fellow scholars and researchers. 


\section{Table of Contents}

Declaration $\quad$ i

Approval ii

Ethics Statement iii

Abstract iv

Dedication $\quad$ v

Acknowledgment vi vi

Table of Contents vii

List of Figures $\quad$ x

List of Tables $\quad$ xi

Nomenclature xii

1 Introduction $\quad 1$

1.1 Background . . . . . . . . . . . . . . . . . 1

1.2 Literature Reviews . . . . . . . . . . . . . . . . . . . . 1

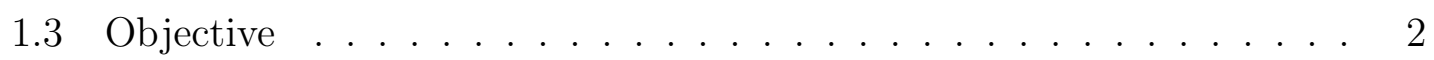

1.4 Overview of Contents . . . . . . . . . . . . . . . . 3

2 Fundamentals of CNN, Image Processing and Brain in Human Body 4

2.1 Convolution Neural Network . . . . . . . . . . . . . . . . . . . . 4

2.2 How Convolutional Neural Networks Work . . . . . . . . . . . . 5

2.2 .1 Input image . . . . . . . . . . . . . . . 5

2.2 .2 Convolution Layer . . . . . . . . . . . . . . . . 5

2.2 .3 Padding . . . . . . . . . . . . . . . . 7

2.2.4 Non Linearity (ReLU) . . . . . . . . . . . . . . . 7

2.2 .5 Pooling Layer . . . . . . . . . . . . . . . . . . . . . . . . . 7

2.2 .6 Fully Connected Layer . . . . . . . . . . . . . . . . . . . . . . 8

2.3 Image Processing . . . . . . . . . . . . . . . . . . . . . . . . 9

2.3.1 Low-Level Image Processing . . . . . . . . . . . . . . . . . . . 10

2.3.2 Mid-Level Image Processing . . . . . . . . . . . . . . . . . . . 10

2.3.3 High-Level Image Processing . . . . . . . . . . . . . . . . . 10 
2.4 Stages of Digital Image Processing _ . . . . . . . . . . . . . 10

2.4.1 Image Acquisition . . . . . . . . . . . . . . . . . . . 11

2.4 .2 Image Enhancement . . . . . . . . . . . . . . . . . 11

2.4.3 Image Restoration . . . . . . . . . . . . . . . . . . . . 12

2.4.4 Color Image Processing . . . . . . . . . . . . . . . . . . . . . . 12

2.4.5 Wavelets and Multiresolution Processing . . . . . . . . . . . . 12

2.4.6 Compression . . . . . . . . . . . . . . . . . 12

2.4.7 Morphological Image Processing . . . . . . . . . . . . . . . 12

2.4.8 Segmentation . . . . . . . . . . . . . . . . . 13

2.4 .9 Representation and Description . . . . . . . . . . . . . 14

2.4 .10 Object Recognition . . . . . . . . . . . . . . . . . . 14

2.4.11 Knowledge Base . . . . . . . . . . . . . . . . . . . . 14

2.5 Brain . . . . . . . . . . . . . . . . . . . . . . . 14

2.5.1 Cerebrum . . . . . . . . . . . . . . 15

2.5.2 Cerebellum . . . . . . . . . . . . . . 15

2.5.3 Brain stem . . . . . . . . . . . . . . . 16

2.5.4 Lobes of the Brain . . . . . . . . . . . . . . 16

2.6 Brain tumor . . . . . . . . . . . . . . . . . 17

2.6.1 Primary Tumors . . . . . . . . . . . . . . . 17

2.6.2 Secondary Tumors . . . . . . . . . . . . . . . 18

2.6.3 Magnetic Resonance Imaging (MRI) . . . . . . . . . . . . . . 18

2.7 Interpolation . . . . . . . . . . . . . . . . . . 18

3 Proposed Method 20

3.1 DataSet Preparation . . . . . . . . . . . . . . . . . 20

3.2 Implementation of Convolution Neural Network . . . . . . . . . . . 20

3.2 .1 Input Image Layer . . . . . . . . . . . . . . . . . 23

3.2 .2 Convolution Layer . . . . . . . . . . . . . . . . . . . . 23

3.2 .3 Stride and Padding . . . . . . . . . . . . . . . . . . 24

3.2 .4 Relu Layer . . . . . . . . . . . . . . . . . . . . . . . . . . . 24

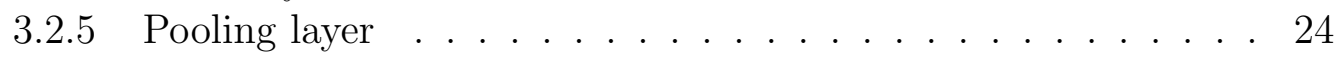

3.2 .6 Flattened . . . . . . . . . . . . . . . . 25

3.2.7 Fully Connected Layer . . . . . . . . . . . . . . . . . . . . 25

3.2 .8 Classification Layer . . . . . . . . . . . . . . . . . 25

3.2 .9 Training Algorithm . . . . . . . . . . . . . . . . . 25

3.3 Artifacts Remove . . . . . . . . . . . . . . . . . . 26

3.4 Noise Remove . . . . . . . . . . . . . . . . . . . . . . . . 26

3.4.1 Median Filter . . . . . . . . . . . . . . . . 26

3.4 .2 Average Filter . . . . . . . . . . . . . . . . . . . 27

3.4 .3 Gauss Filter . . . . . . . . . . . . . . . . 27

3.4.4 Un-sharped Masking Filter . . . . . . . . . . . . . . . . . 27

3.5 Comparison Parameter . . . . . . . . . . . . . . . . . . 27

3.5.1 Segmentation . . . . . . . . . . . . . 28

4 Experiments and Results $\quad 31$

4.1 Accuracy Measurement . . . . . . . . . . . . . . . . . 31

4.2 Image Pre-processing . . . . . . . . . . . . . . . . . . . . . . . 32

4.2.1 Artifacts Remove . . . . . . . . . . . . . . . . . . . 32

4.2 .2 Noise Remove . . . . . . . . . . . . . . . . . . . . 33 
4.2 .3 Tumor Segmentation . . . . . . . . . . . . . . . 36

4.2 .4 3D Visualization . . . . . . . . . . . . . . . . 38

5 Conclusion and Future Works 40

5.1 Concluding Remark . . . . . . . . . . . . . . . . . . . . . . . . . . . 40

5.2 Future Works . . . . . . . . . . . . . . . . . . . . 40

$\begin{array}{ll}\text { Bibliography } & 43\end{array}$

Appendix A How to install $\mathrm{HT}_{\mathrm{E}} \mathrm{X}$

Appendix B Overleaf: GitHub for $\mathrm{HT}_{\mathrm{E}} \mathrm{X}$ projects $\quad 47$ 


\section{List of Figures}

1.1 Different Types of MRI Images . . . . . . . . . . . . . . . . . 1

2.1 A CNN sequence to classify handwritten digits . . . . . . . . . . 5

2.2 RGB 3 Channels . . . . . . . . . . . . . . . . . 6

2.3 Convoluting a $5 \times 5 \times 1$ image with a $3 \times 3 \times 1$ kernel to get a $3 \times 3 \times 1$ convolved feature ................... . . . 6

2.4 Types of Pooling . . . . . . . . . . . . . . . . . 8

2.5 After pooling layer, flattened as FC layer . . . . . . . . . . . . . . . . 9

2.6 Steps in digital image processing . . . . . . . . . . . . . . . 11

2.7 Image Acquisition Model . . . . . . . . . . . . . . . . . . . . 11

2.8 Brain Of Human Body . . . . . . . . . . . . . . . . . . . . 15

2.9 Diagram of a MRI Scanner . . . . . . . . . . . . . . . 18

2.10 Interpolation Algorithm . . . . . . . . . . . . . . . . . 19

3.1 Work flow of Proposed Model. . . . . . . . . . . . . . . . . . . . . . . 21

3.2 Implementation of Convolution Neural Network. . . . . . . . . . . . . 22

3.3 Structuring Element. . . . . . . . . . . . . . . . 26

3.4 Process diagram of edge-detection by multi-level thresholding. . . . . 29

4.1 Confusion Matrix. . . . . . . . . . . . . . . . . . . . 31

4.2 Artifacts removal results using different pixel value. . . . . . . . . . . 33

4.3 Noise removal results using different filters. . . . . . . . . . . . . . . . 34

4.4 Overview of the result of PSNR and MSE on T1. . . . . . . . . . . 35

4.5 Overview of the result of PSNR and MSE on T2. . . . . . . . . . . 35

4.6 Overview of the result of PSNR and MSE on Flair. . . . . . . . . . . 35

4.7 Image Segmentation. . . . . . . . . . . . . . . . . . . . . . . . . . . . . . . . . . 37

4.8 Axial View of Tumor. . . . . . . . . . . . . . . . . . . . . 38

4.9 Segital Section (on Left) Cornal Section (on Right). . . . . . . . . . 39

4.10 3D Visualization of Identified Tumor. . . . . . . . . . . . . . . . . 39 


\section{List of Tables}

4.1 The average PSNR and MSE results . . . . . . . . . . . . 36 


\section{Nomenclature}

The next list describes several symbols \& abbreviation that will be later used within the body of the document

IPL Indian Premier League

$L B W$ Leg before Wicket

$M R \quad$ Runs scored by Home team

$M R N$ Home Team Run Rate

$O D I$ One day International

OR Runs scored by the opponent team

ORN Opponent Team Run Rate

T20 Twenty Twenty

CNN Convulational Neural Network 


\section{Chapter 1}

\section{Introduction}

Magnetic resonance imaging $(\mathrm{MRl})$ has become essential part of medical science. It hasseveral advantages over other medical imaging modalities with many applications, such as,cardiovascular, neurological, and in particular brain imaging. From last decade MRI imagesare become more popular to identify the tumors from brain [4].

\subsection{Background}

Magnetic resonance imaging (MRI), Nuclear Magnetic Resonance Imaging (NMRI), or Magnetic Resonance Tomography (MRT) are medical imaging technique used in radiology to visualize internal structures of the body. Comparing on other medical imaging technique, MRI gives detail study of human body. On the other hand, MRI images does not produce any kind of harmful radiation. MRI gives good contract of soft tissue of human body [3]. As brain is consists of soft tissue, MRI is used for finding any diseases of brain such as cancer and tumor etc.

There are two types of MRI images such as T1 and T2 weighted image, FLAIR and MRS images. MRI images can be classified into three regions such as Grey matter (GM), White matter (WM) and Cerebrospinal Fluid (CSF). In T1 weighted image, White matter is brighter than Gray matter. On the other hand, in T2 weighted image Gray matter is lighter than White matter [17].
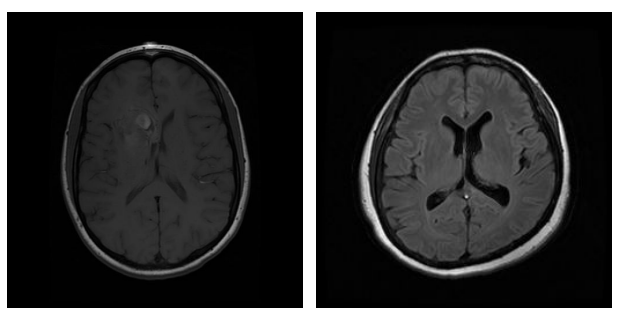

Figure 1.1: T1 weighted image (on left) 85 T2 weighted image (on right).

\subsection{Literature Reviews}

Chaddad, Zinn and Colen presented multi thresholding segmentation with morphological operations in their paper of MR images for detecting abnormal area of brain. 
After that they used Gaussian Mixture Model (GMM) to extract feature of brain tumor. Finally, Decision Tree classifier is applied on GMM features to evaluate the performance of cancer and normal area discrimination [4].

Saravanan, Vishnuvarthanan, Pallikonda Rajasekaran applied tree matrix graph cutting (TM) algorithm for brain image segmentation and identification which has three sequence steps. In the first step, they applied pre-processing which generates tree of labels as key to the tree matrix algorithm. Globally optimal labeling was identified in the second step with respect to the tree of labels. Finally, in the post-processing step, they used "tree-cutting" method to generate a mapping from labels to brain tissues such as Grey Matter (GM), White Matter (WM) and Cerebrospinal Fluid (CSF) producing a meaningful MR brain image segmentation [14].

Ramya and Grace used watershed transformation technique with morphological open image and two important features is used as foreground and background to identify the tumor. They used gradient magnitude with morphological open image. With the gradient magnitude for segmentation technique the rate of inclination or declination of a tumor will be identified [18].

Moftah, Ghali, Hassanien and Ismail basically sliced whole MRI images with small pieces and then clustered and labeled of brain tumor for every sliced. This paper deals with two dimensional magnetic resonance imaging (MRI) sequence of brain slices which includes many objects to identify and estimate the volume of the brain tumors. Twenty-five or more highlights dependent on shape, shading and surface was extricated to acquire include vector for each object to identify the tumor and recognize it [7].

Wang Mengqiao, Yang Jie, Chen Yilei and Wang Hao used Convolutional Neural network for the challenging problem of gliomas segmentation. To reduce overfitting they used add dropout and speed up training with the help of batch normalization technique. Moreover, to correct the bias field distortion of MRI images, they have added N4ITK method before intensity normalization to get the actual result [34]. Md. Rokibul Islam and Nafees Rishad gave some direction to determine effective values for CNN filter size and number in Brain MRI image classification. For better accuracy in classification they found that square filters are better than rectangular filter; larger number of filters produces more complexities with higher classification accuracy and increasing of convolutional stride results decrease in network accuracy [33].

R.Vinoth and Chunchu Venkatesh used CNN for classification. By calculating certain parameters of the image they have completed their extension work. After completing the calculation they found the depth of infection. Then they used SVM classification with the calculated parameters to get the actual result [28].

\subsection{Objective}

The objective of this research study is to classification, segmentation of brain tumor and finally 3D representation of affected area of tumor visually. Nowadays, health care system mostly depends on medical image processing for extracting information about human body to diagnose almost all diseases. This research paper gives a methodology to identify and feature extraction affected tumor of brain. At the end of the paper of we show the 3D visualization of affected area of tumor in brain so that before starting actual treatment doctors will get a general idea how to proceed. 
We believe, our proposed method will provide more accurate result in the way to detect as well as this will make the process simple than the more complex analysis of the brain tumor. We believe that after this research comes into physical being, dependency on human visual inspection will be highly minimized and more accurate diagnosis will be ensured.

\subsection{Overview of Contents}

The remainder of this paper is organized as follows,

- Chapter 2 : Briefly reviews of fundamentals of CNN, Image Processing and Brain in Human Body.

- Chapter 3 : Explanation of our proposed methods.

- Chapter 4: Experimental results and discussions based on a comparative study.

- Chapter 5 : Conclusion and Future Works.

- Chapter 6 : References. 


\section{Chapter 2}

\section{Fundamentals of CNN, Image Processing and Brain in Human Body}

In this chapter, we discussed about, fundamentals of image processing and different parts brains of human body.

\subsection{Convolution Neural Network}

Artificial Intelligence has been witnessing a monumental growth in bridging the gap between the capabilities of humans and machines. Researchers and enthusiasts alike, work on numerous aspects of the field to make amazing things happen. One of many such areas is the domain of Computer Vision.

The agenda for this field is to enable machines to view the world as humans do, perceive it in a similar manner and even use the knowledge for a multitude of tasks such as Image \& Video recognition, Image Analysis Classification, Media Recreation, Recommendation Systems, Natural Language Processing etc. The advancements in Computer Vision with Deep Learning has been constructed and perfected with time, primarily over one particular algorithm - a Convolutional Neural Network Convolutional neural networks are deep artificial neural networks that are used primarily to classify images (e.g. name what they see), cluster them by similarity (photo search), and perform object recognition within scenes. They are algorithms that can identify faces, individuals, street signs, tumors, platypuses and many other aspects of visual data [20].

Convolutional networks perform optical character recognition (OCR) to digitize text and make natural-language processing possible on analog and hand-written documents, where the images are symbols to be transcribed. CNN can also be applied to sound when it is represented visually as a spectrogram. More recently, convolutional networks have been applied directly to text analytics as well as graph data with graph convolutional networks. [4]

The efficacy of convolutional nets (ConvNets or CNN) in image recognition is one of the main reasons why the world has woken up to the efficacy of deep learning. They are powering major advances in computer vision (CV), which has obvious applications for self-driving cars, robotics, drones, security, medical diagnoses, and treatments for the visually impaired. 
A ConvNet is able to successfully capture the Spatial and Temporal dependencies in an image through the application of relevant filters. The architecture performs a better fitting to the image dataset due to the reduction in the number of parameters involved and reusability of weights. In other words, the network can be trained to understand the sophistication of the image better.

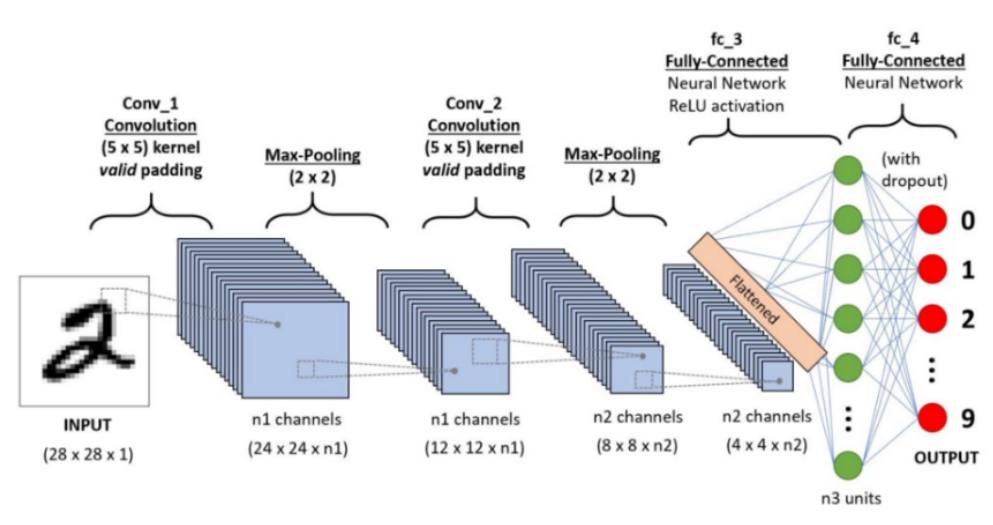

Figure 2.1: A CNN sequence to classify handwritten digits [30].

\subsection{How Convolutional Neural Networks Work}

CNNs, like neural networks, are made up of neurons with learnable weights and biases. Each neuron receives several inputs, takes a weighted sum over them, pass it through an activation function and responds with an output.

\subsubsection{Input image}

In the figure, we have an RGB image which has been separated by its three color planes - Red, Green, and Blue. There are a number of such color spaces in which images exist-Grayscale, RGB, HSV, CMYK, etc.You can imagine how computationally intensive things would get once the images reach dimensions, say $8 \mathrm{~K}$ (76804320). The role of the ConvNet is to reduce the images into a form which is easier to process, without losing features which are critical for getting a good prediction. This is important when we are to design an architecture which is not only good at learning features but also is scalable to massive datasets.

\subsubsection{Convolution Layer}

Convolution is the first layer to extract features from an input image. Convolution preserves the relationship between pixels by learning image features using small squares of input data. It is a mathematical operation that takes two inputs such as image matrix and a filter or kernel. 


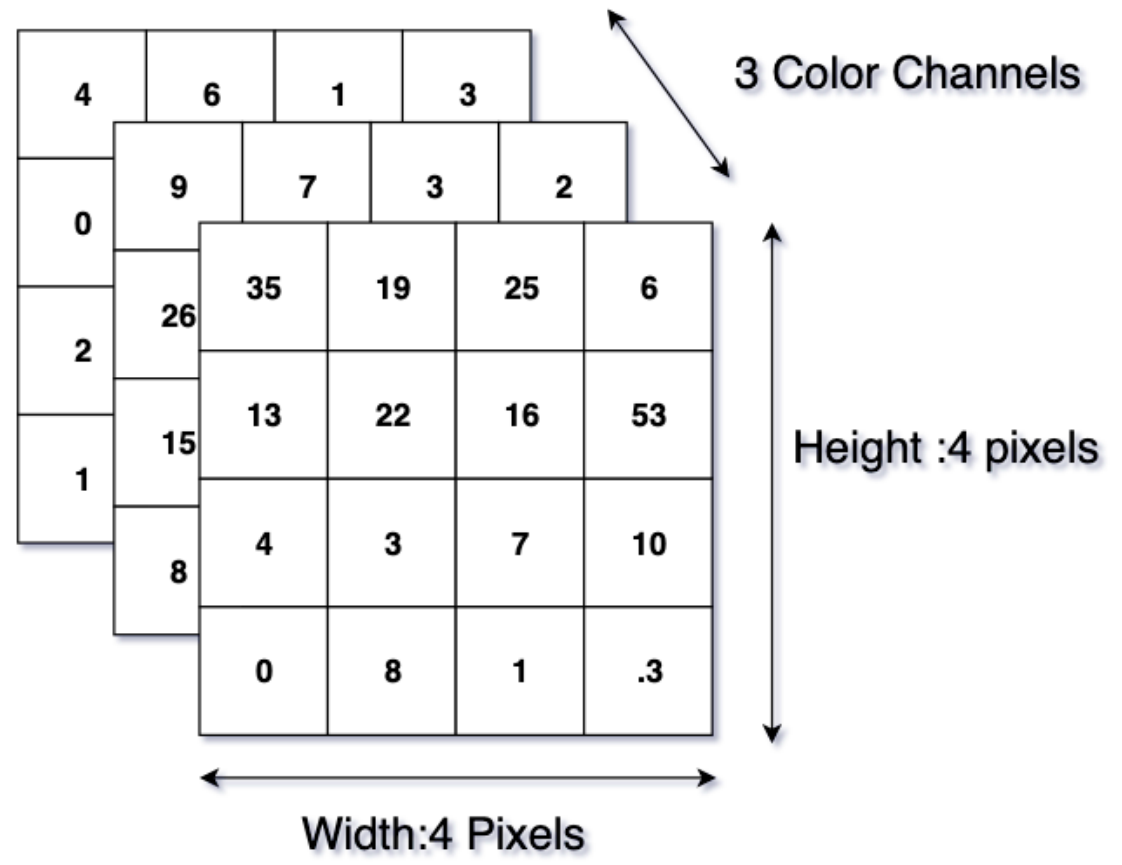

Figure 2.2: RGB 3 Channels.
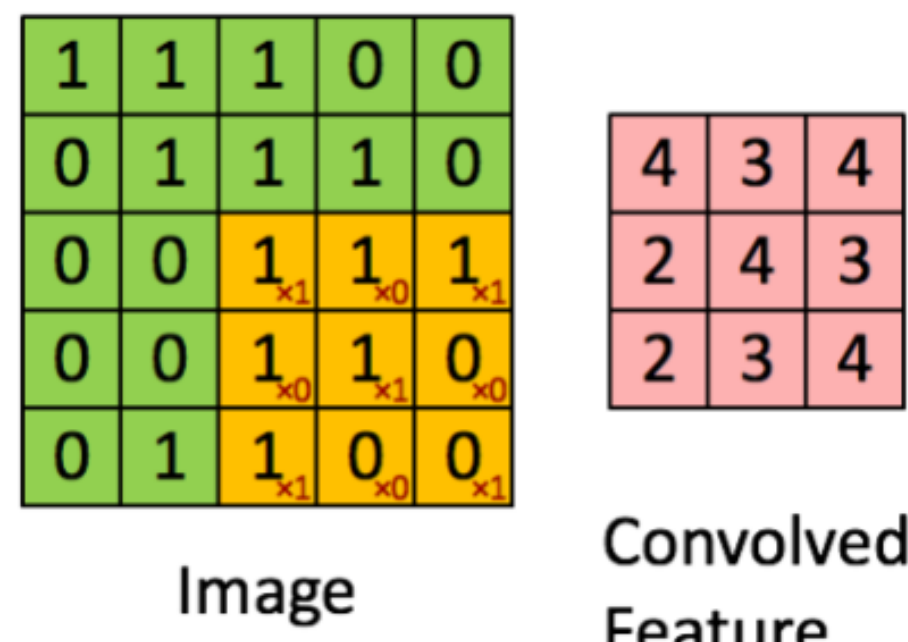

Convolved Feature

Figure 2.3: Convoluting a 5x5x1 image with a 3x3x1 kernel to get a 3x3x1 convolved feature. 
Image Dimensions $=5$ (Height) $\times 5$ (Breadth) $\times 1$ (Number of channels, eg. RGB).In the above demonstration, the green section resembles our 5x5x1 input image, I. The element involved in carrying out the convolution operation in the first part of a Convolutional Layer is called the Kernel/Filter, K, represented in the color yellow. We have selected $\mathrm{K}$ as a 3x3x1 matrix.The Kernel shifts 9 times because of Stride Length $=1$ (Non-Stride), every time performing a matrix multiplication operation between $\mathrm{K}$ and the portion $\mathrm{P}$ of the image over which the kernel is hovering [8].

\subsubsection{Padding}

The objective of the Convolution Operation is to extract the high-level features such as edges, from the input image. ConvNets need not be limited to only one Convolutional Layer. Conventionally, the first ConvLayer is responsible for capturing the Low-Level features such as edges, color, gradient orientation, etc. With added layers, the architecture adapts to the High-Level features as well, giving us a network which has the wholesome understanding of images in the dataset, similar to how we would. There are two types of results to the operation-one in which the convolved feature is reduced in dimensionality as compared to the input, and the other in which the dimensionality is either increased or remains the same.

\section{Same Padding}

When we augment the 5x5x1 image into a $6 \times 6 \times 1$ image and then apply the $3 \times 3 \times 1$ kernel over it, we find that the convolved matrix turns out to be of dimensions $5 \times 5 \times 1$.

\section{Valid Padding}

We perform the same operation without padding, we are presented with a matrix which has dimensions of the Kernel $(3 \times 3 \times 1)$ itself

\subsubsection{Non Linearity (ReLU)}

ReLU stands for Rectified Linear Unit for a non-linear operation. The output is (x) $=\max (0, \mathrm{x})$. ReLU's purpose is to introduce non-linearity in our ConvNet. Since the real world data would want our ConvNet to learn would be non-negative linear values. There are other nonlinear functions such as tanh or sigmoid can also be used instead of ReLU. Most of the data scientists use ReLU since performance wise ReLU is better than the other two [10].

\subsubsection{Pooling Layer}

The Pooling layer is responsible for reducing the spatial size of the Convolved Feature. This is to decrease the computational power required to process the data through dimensionality reduction. Furthermore, it is useful for extracting dominant features which are rotational and positional invariant, thus maintaining the process of effectively training of the model.

There are two types of Pooling: Max Pooling and Average Pooling. Max Pooling returns the maximum value from the portion of the image covered by the Kernel. 
On the other hand, Average Pooling returns the average of all the values from the portion of the image covered by the Kernel.

Max Pooling also performs as a Noise Suppressant. It discards the noisy activations altogether and also performs de-noising along with dimensionality reduction. On the other hand, Average Pooling simply performs dimensionality reduction as a noise suppressing mechanism. Hence, we can say that Max Pooling performs a lot better than Average Pooling.The Convolutional Layer and the Pooling Layer, together form the i-th layer of a Convolutional Neural Network.Depending on the complexities in the images, the number of such layers may be increased for capturing low-levels details even further, but at the cost of more computational power.

After going through the above process, we have successfully enabled the model to understand the features. Moving on, we are going to flatten the final output and feed it to a regular Neural Network for classification purposes.

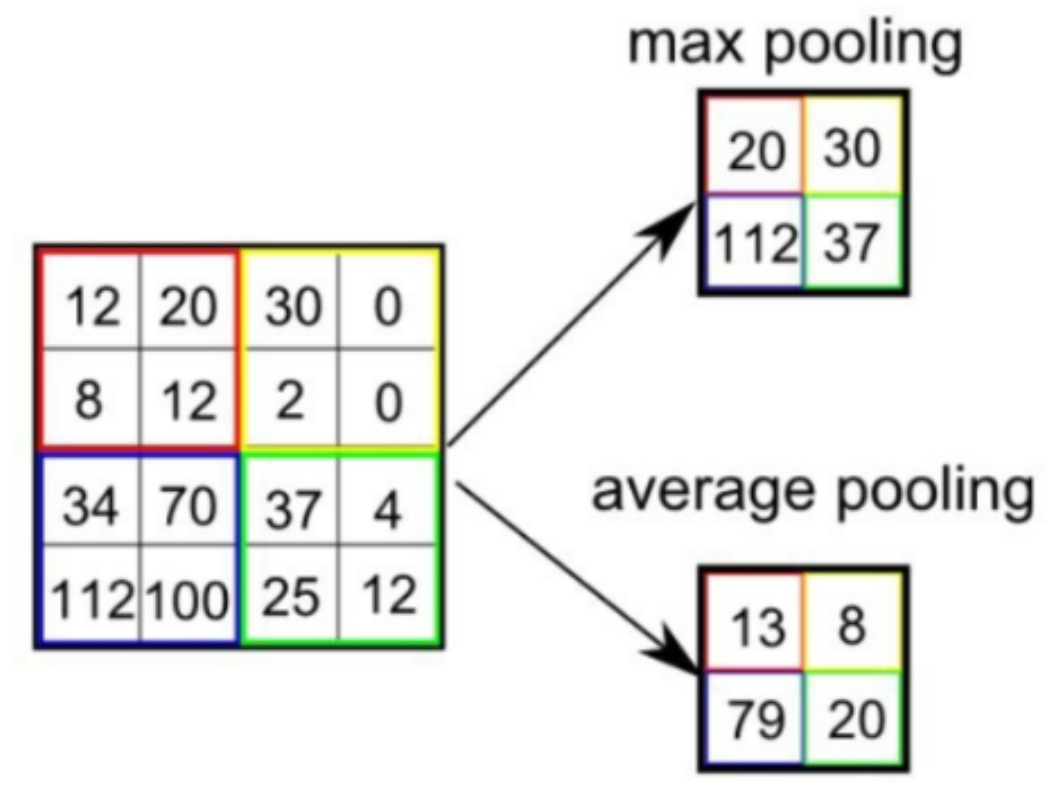

Figure 2.4: Types of Pooling.

\subsubsection{Fully Connected Layer}

The layer we call as FC layer, we flattened our matrix into a vector and feed it into a fully connected layer like a neural network.

Now that we have converted our input image into a suitable form for our MultiLevel Perceptron, we shall flatten the image into a column vector. The flattened output is fed to a feed-forward neural network and backpropagation applied to every iteration of training. Over a series of epochs, the model is able to distinguish between dominating and certain low-level features in images and classify them using the Softmax Classification technique [32].

There are various architectures of CNNs available which have been key in building algorithms which power and shall power AI as a whole in the foreseeable future. Some of them have been listed below:

- LeNet 
- AlexNet

- VGGNet

- GoogLeNet

- ResNet

- ZFNet

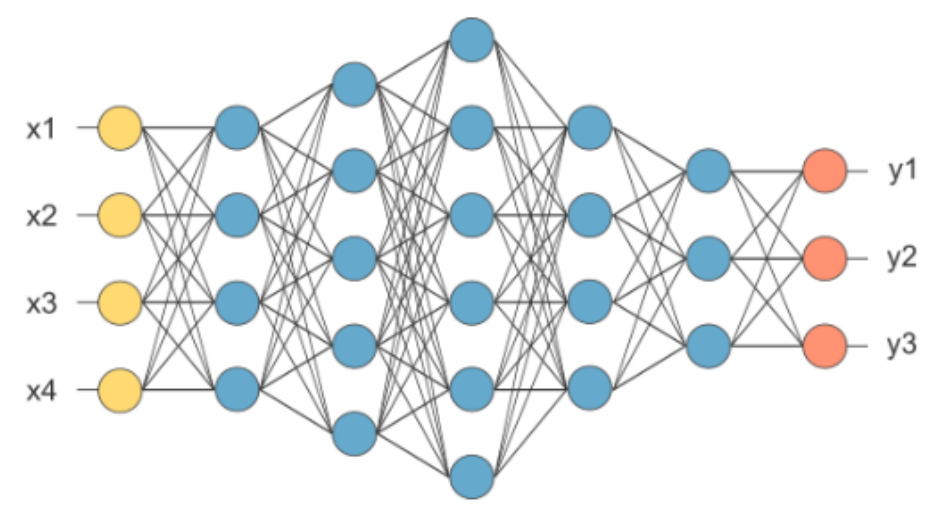

Figure 2.5: After pooling layer, flattened as FC layer [32].

\subsection{Image Processing}

Image processing is a method to convert an image into digital form and perform some operations on it, in order to get an enhanced image or to extract some useful information from it. It is a type of signal dispensation in which input is image, like video frame or photograph and output may be image or characteristics associated with that image [4].

Image processing was first used in the early 1920s in a paper industry where images were coded for a submarine cable transfer reconstructed by a telegraph printer at the receiving point. In the mid to late 1920 s, there had been improvements in the system. In 1964, image processing was used to improve the images of the moon taken by the Ranger 7 space probe [14]. Such techniques were used in the other space missions as well. In the 1970s, image processing began to be used in the fields of medical science. In 1979, Allan M. Cormack and Godfrey N. Hounsfield jointly received The Nobel Prize for the invention of computer-assisted tomography.

An image is an artifact that depicts visual perception, for example, a photo or a twodimensional picture, which has a similar appearance to some subject. An image may be defined as a two-dimensional function, $\mathrm{f}(\mathrm{x}, \mathrm{y})$, where $\mathrm{x}$ and $\mathrm{y}$ are spatial (plane) coordinate, and amplitude of at any pair of coordinates $(\mathrm{x}, \mathrm{y})$ is called the intensity or gray level of the image at that point. When $\mathrm{x}, \mathrm{y}$ and the amplitude values of $\mathrm{f}$ are all finite, discrete quantities, we call the image a digital image. A digital image is composed of a finite number of elements, each of which has a particular location and values. These elements are referred to as picture elements, image elements and pixels. Pixels are the term most widely used to denote the element of digital image 
[5].

Image processing includes the following three steps:

- Importing the image with optical scanner or by digital photography.

- Analyzing and manipulating the image, which includes data compression and image enhancement, and spotting patterns that are not to human eyes like satellite photographs.

- Output is the last stage in which result can be altered image or report that is based on image analysis.

There are two types of methods used for image processing namely, analogue and digital image processing. Analogue image processing can be used for the hard copies like prints and photographs. Image analysts use various fundamentals of interpretation while using these visual techniques [31]. Digital image processing techniques help in manipulation of the digital images by using computers. Images go through for digital image processing in three phases:

- Low-level image processing

- Mid-level image processing

- High-level image processing

\subsubsection{Low-Level Image Processing}

Main purpose of this phase is noise-reduction, image-sharpening, enhancement etc.

\subsubsection{Mid-Level Image Processing}

Image analysis phase where segmentation and classification of image operation is carried out.

\subsubsection{High-Level Image Processing}

The final phase where we can visualize the output image by assembling the recognized objects.

\subsection{Stages of Digital Image Processing}

Image processing is a series of process and the output is acquired by systematic execution of several operations.Some core stages that is common is all sort of image processing are stated below: 


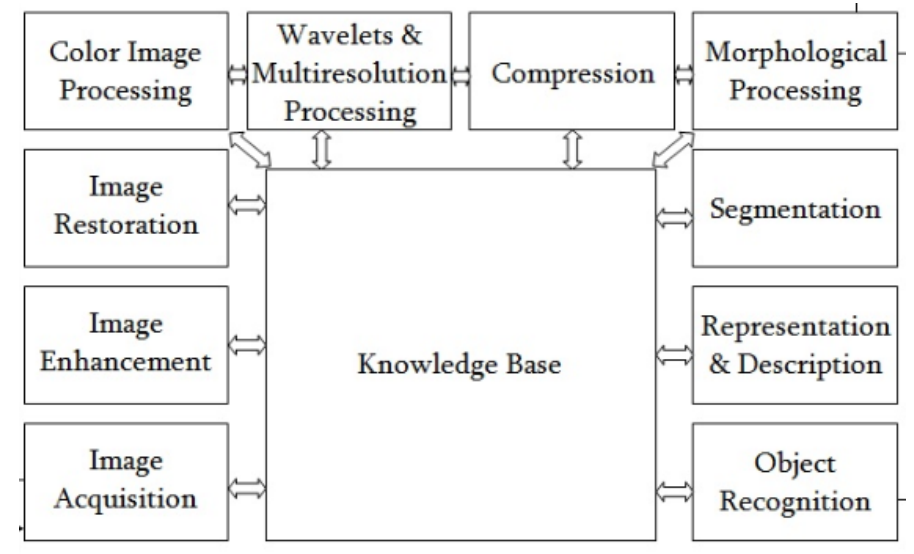

Figure 2.6: Steps in digital image processing [7].

\subsubsection{Image Acquisition}

This is the first step or process of the fundamental steps of digital image processing. The images are generated by combination of an illumination source and the reflection or absorption of the energy by the elements of scene being imaged[18] .To sense the image, we use sensor according to the nature of illumination. The process of image sense is called image acquisition.Generally, the image acquisition stage involves pre-processing, such as scaling etc.

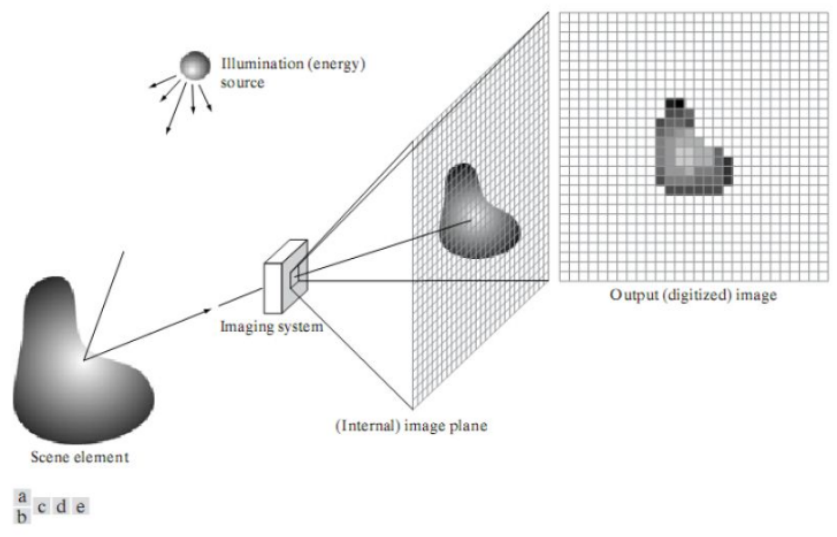

Figure 2.7: Image Acquisition Model [31].

\subsubsection{Image Enhancement}

Image enhancement is the simplest and most appealing areas of digital image processing.Image Enhancement is the process of manipulating an image so that the result is more suitable than the original for specific applications Enhancement simply to highlight certain features of interest in an image. It is a very subjective area of image processing.Enhancement techniques are so varied, and use so many different image processing approaches. 


\subsubsection{Image Restoration}

Image restoration is an area that also deals with improving the appearance of an image. Image restoration is objective, the sense that restoration techniques tend to be based on mathematical or probabilistic model of image degradation [24]. A simple formula of the image restoration is:

$$
y(i, j)=H[f(i, j)]+n(i, j)
$$

Here, $y(i, j)$ is the degraded image, $f(i, j)$ is the original image, $H$ is the operator for degradation process and $n(i, j)$ is the external noise.

\subsubsection{Color Image Processing}

Nowadays color image processing is an area that has been gaining in importance because of the significant increase in the use of digital images over the Internet. Color is used for extracting the features of interest in an image.

\subsubsection{Wavelets and Multiresolution Processing}

Wavelets are the foundation for representing images in various degree of resolution, in which images are subdivided successfully into smaller regions [1]. Multiresolution analysis is a representation of a signal in more than one resolution/scale.

\subsubsection{Compression}

Image compression deals with two major techniques :

1. Reducing the storage required to save an image.

2. Reducing the size of the image to transmit it ("JPEG Standard"), with suitable bandwidth required for transmission.

\subsubsection{Morphological Image Processing}

Morphological processing deals with tools for extracting image component that is useful in the representation and description of shape.Morphological image processing is a collection of non-linear operations related to the shape or morphology of features in an image.Morphological operations rely only on the relative ordering of pixel values, not on their numerical values, and therefore are especially suited to the processing of binary images. Morphological operations can also be applied to grayscale images such that their light transfer functions are unknown and therefore their absolute pixel values are of no or minor interest [11].

Morphological techniques probe an image with a small shape or template called a structuring element. The structuring element is positioned at all possible locations in the image and it is compared with the corresponding neighbourhood of pixels. The structuring element is a small binary image small matrix of pixels, each with a value of zero or one. 


\section{Dilation}

In dilation, pixels are added to the boundary of the object. Number of pixels which will be added depends on the size of the object and shape of the structuring element. For binary images, pixels are set 0 . On the other hand, for gray scale images, the minimum value is 0 for uint8 images.

\section{Erosion}

In erosion, pixels are removed from the boundary of the object. Number of pixels which will be removed depends on the size of the object and shape of the structuring element. For binary images, pixels are set 1 . On the other hand, for gray scale images, the minimum value is 1 for uint8 images.

\section{Opening}

Opening operation removes small objects in the background of any image. Basically, it takes the bright pixels of the images foreground. These methods can be described by the following equation,

$$
A \circ B=(A \ominus B) \oplus B
$$

Here $\oplus$ and $\ominus$ indicate dilation and erosion, respectively [29].

\section{Closing}

When processing the images, closing operation removes small holes of any image.

$$
A \circ B=(A \oplus B) \ominus B
$$

Here $\oplus$ and $\ominus$ indicate dilation and erosion, respectively [29].

\subsubsection{Segmentation}

Segmentation producers partition an image into its constituent part or objects. Segmentation is one of the most difficult tasks in digital image processing. Segmentation procedure brings the process a long way toward successful solution of imaging problems that require object to be identified individually [22].Segmentation can be classified into the following types:

- Region based.

- Edge based.

- Threshold.

- Feature based clustering and

- Model based. 


\subsubsection{Representation and Description}

Representation and description always follows the output data from segmentation stage, which is raw pixel data. Representation makes a decision whether the data should be represented as a boundary or as a complete region. Boundary Representation focuses on external shape characteristics, such as corners and inflections.Region Representation focuses on internal properties, such as texture or skeleton shape.Description is also known as feature selection, deals with extracting attributes that results in some quantitative information of interest or are basic for differentiating one class from another.

\subsubsection{Object Recognition}

Recognition is the process that assigns label to an object based on the information provided by its descriptors. Like human understanding, it includes:

- Detection

- Description

- Classification

- Identification and

- Understanding

\subsubsection{Knowledge Base}

The Knowledge base also controls the interaction between modules. The knowledge about a problem is coded into an image processing system in the form of a Knowledge base.

\subsection{Brain}

The brain is a stunning three-pound organ that controls all elements of the body, deciphers data from the outside world, and exemplifies the quintessence of the brain and soul. Insight, inventiveness, feeling, and memory are a couple of the numerous things represented by the cerebrum. The brain is made out of the cerebrum, cerebellum, and brainstem.

The brain gets data through our five detects sight, smell, contact, taste, and hearing - regularly numerous at one time. It gathers the messages in a way that has importance for us, and can store that data in our memory. The cerebrum controls our considerations, memory and discourse, development of the arms and legs, and the capacity of numerous organs inside our body brain1952neurological

The Central Nervous System (CNS) is made out of the cerebrum and spinal rope. The Peripheral Nervous System (PNS) is made out of spinal nerves that branch from the spinal cord and cranial nerves that branch from the brain. The brain is composed of the cerebrum, cerebellum, and brainstem. 


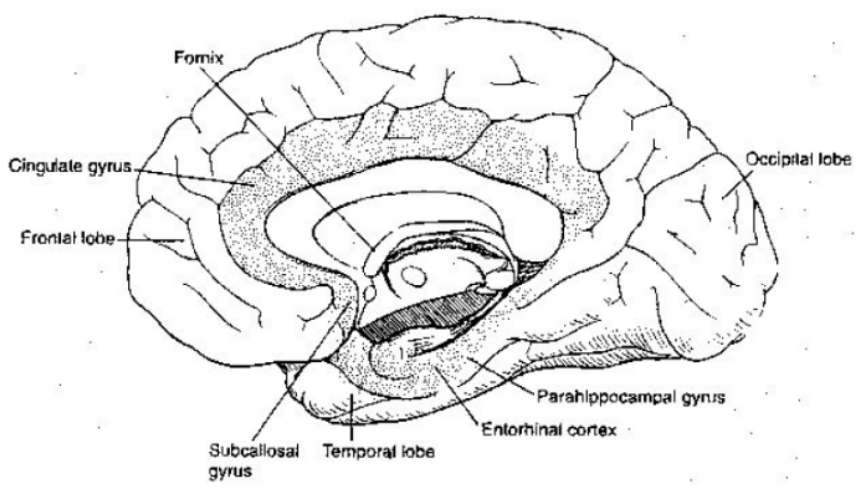

Figure 2.8: Brain Of Human Body [10].

\subsubsection{Cerebrum}

The largest part of the brain and is composed of right and left hemispheres. It performs higher functions like interpreting touch, vision and hearing, as well as speech, reasoning, emotions, learning, and fine control of movement. The cerebral cortex is made of nerve fibers which is called gray matter. On the other hand, the inner layer is made of different types of nerve fibers which is known as white matters. Moreover, the basal ganglia are found in the white matter. The cerebrum is divided in to left and right hemispheres. The left side controls the right side of the body and the left side of the body is controls by the right half of the brain. The cerebral cortex is the outer layer of the brain, which is called gray matter. Many of the signals our brain receives from our senses are registered in the cerebral cortex. The visual cortex is in the lower back part of the brain and is where our brain registers what we see. The somatosensory cortex is a band that runs over the top of the brain is where our brain registers a touch on any part of our body. The motor cortex is just in front of the somatosensory cortex and it sends out signals to muscles to make them move. Moreover, the nerve endings a part of the body has, the more of the sensory cortex it occupies. A big portion of the sensory cortex is taken up by our lips and face.

\subsubsection{Cerebellum}

The cerebellum is known as little brain. It is situated behind and underneath the cerebrum and at the back of the brain stem and connected to the midbrain. It has two sides of the equator and an external cortex of gray matter and an internal core of white matter. The cerebellum is associated with development and coordination, strolling, pose, reflexes, eye and head development. It organizes intuitive developments, for example, balance and facilitated development. The cerebellum is always getting updates about the body's position and development. It additionally sends guidelines to our muscles that modify our stance and keep our body moving easily. The diencephalon is situated between the cerebrum and midbrain. It comprises of the thalamus and nerve center which lie somewhere down in the cerebral sides of the hemispheres. Focuses in the nerve center manage our body temperature, glucose, hunger and hormones. The thalamus is included with tactile signs sent to the higher forebrain, specifically the cerebral cortex. The thalamus additionally takes an interest in engine control and managing cortex energy. A few pathways associate 
the brainstem to the lower engine focuses in the spinal line and the higher ones in the forebrain.

\subsubsection{Brain stem}

Brain stem acts as a relay center connecting the cerebrum and cerebellum to the spinal cord. It performs many automatic functions such as breathing, heart rate, body temperature, wake and sleep cycles, digestion, sneezing, coughing, vomiting, and swallowing. It consists of midbrain, pons and medulla oblongata. Moreover, it is also known as body's autopilot. It shows the pathways for nerve fibers between the higher and lower neural centers.

\section{Midbrain}

Mid brain situated at the base of the brain and small but important region which serves as important connection point between the forebrain and the hindbrain. The midbrain is located in the upper part of the brainstem. Moreover, the connection central between the brain and the spinal code. Moreover, it is the reflex center for cranial nerves III (oculomotor-moves eye, pupil) and IV (trochlear- moves eye).

\section{Pons}

Pons is situated beside the medulla oblongata. It is the major structure in the upper part of brainstem. It helps regular breathing. Moreover, it connects the cerebellum with the cerebrum and links the midbrain to the medulla oblongata. However, the pons reflex center for cranial nerves V (trigeminal-face sensation) through VIII (vestibulocochlear-hearing, balance). The pons is involved in chewing, taste, saliva, hearing and equilibrium.

\section{Medulla oblongata}

The medulla oblongata connects the spinal cord at the foramen magnum. Moreover, it works for heart, breathing, and circulation. However, it is known as the center for vomiting, coughing and hiccupping.

\subsubsection{Lobes of the Brain}

The cerebral sides of the equator have particular crevices, which partition the brain into lobes. Every side of the hemispheres has 4 lobes: frontal, temporal, parietal, and occipital (Fig. 2). Every lobe might be partitioned into zones that serve quite certain capacities. It's essential to comprehend that every projection of the mind does not work alone. There are exceptionally intricate connections between the lobes of the brain and between the right and left halves of the hemisphere.

\section{Frontal Lobe}

Frontal lobe controls emotions, behavior and personality. It helps to do judgement, planning and problem solving. Moreover, it helps our body to move, speech, concentrate and self-awareness. 


\section{Parietal Lobe}

It interprets language and words. Parietal lobe signals the body to sense of touch, pain and temperature. Moreover, it includes spatial sense and navigation. The important receptive area for the sense of touch in the somatosensory cortex.

\section{Occipital Lobe}

It plays a vital role in integrating our vision and make sense what the eyes sees.

\section{Temporal Lobe}

It mainly works for hearing and selective listening. It controls our memory system. Moreover, it helps to sequence and organize our work.

\subsection{Brain tumor}

Brain tumor is a mass of abnormal cells in our brain. The skull which cover our brain is very inflexible. The growth of anything inside such a restricted space can cause problems. Moreover, this brain tumor can be malignant(cancerous) or benign (noncancerous). To be more specific, if this cancerous and noncancerous grow, it creates pressure to grow inside the skull [13]. As a result, it causes brain damage and also it can be harmful for a life.Brain tumors are two types. They are primary brain tumor and secondary brain tumor.

\subsubsection{Primary Tumors}

A primary tumor started to grow in the brain. Most of the primary tumors are noncancerous. This primary tumor developed from our brain cells, meninges, nerve cells and glands. The common types of primary brain tumors are gliomas and meningiomas.

\section{Gliomas}

Gliomas tumors develop from glial cells. The glial cells mainly support the structure of our central nervous system. Moreover, it provides nutrition to the nervous system also it cleans the cellular waste. Astrocytic tumors started to grow in cerebrum which is a part of glial cells. Oligodendroglial tumors are found in frontal temporal lobes. This types of tumors started in glial cells. Moreover, there are some other primary brain tumors like pituitary tumors, pineal gland tumors, ependymomas craniopharyngiomas, lymphomas.

\section{Meningioma}

The meningioma attacked people in the age between 40 and 70. It is very common in women rather than men. This tumor is usually non cancerous but it can cause vital issues because of their size and locations. Cancerous meningioma is very rare but if it attacks it will be very harmful. 


\subsubsection{Secondary Tumors}

A secondary brain tumor is known as metastatic brain tumor. It occurs when cancer cells spread to the brain from another part of the organ. It might be spread from lung cancer, breast cancer, kidney cancer and skin cancer. Moreover, secondary tumors are always malignant.

\subsubsection{Magnetic Resonance Imaging (MRI)}

MRI stands for magnetic resonance imaging which is a test that uses magnets, radio waves and a computer to make detailed pictures of internal body parts. It helps to diagnose and help the doctor for proper treatment. However, it does not use radiation like X-rays and $\mathrm{CT}$ scans. Moreover, MRI scanning is a non-invasive and painless procedure [9]. MRI helps to detect anomalies of the brain and spinal cord, tumors, cysts, breast cancer, certain types of heart diseases etc. The filtering activity is controlled from a central PC. This determines the state of slope and r.f. wave forms, and timings to be utilized, and passes this data to the waveform generator, which yields the signs and passes them to be intensified and sent to the loops. The NMR flag, when it has been stage delicately distinguished, is swung to an advanced flag by simple to computerized converter. The computerized flag is then sent to a picture processor for Fourier change and the picture is shown on a screen [2].

The crude information, that is the flag before Fourier change, is put away to empower the use of remedies to the information in post preparing. To permit the utilization of quick Fourier change, network sizes of $2 \mathrm{n}$ are normally utilized.

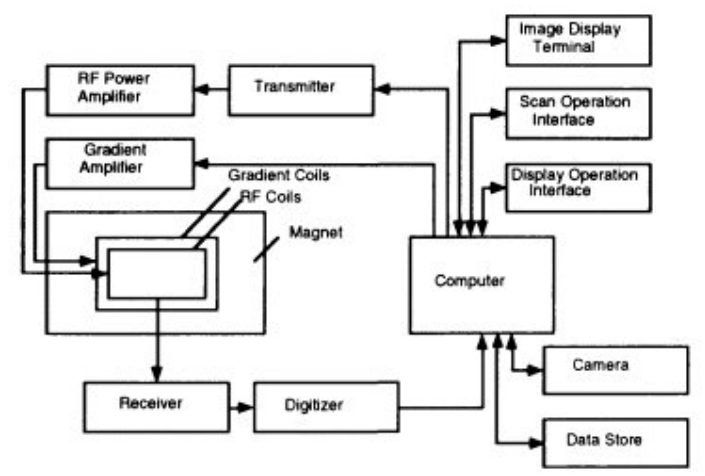

Figure 2.9: Diagram of a MRI Scanner [27].

\subsection{Interpolation}

Interpolation is the process of deriving a simple function from a set of discrete data points so that the function passes through all the given data points and can be used to estimate data points in-between the given ones. It is necessary because in science and engineering we often need to deal with discrete experimental data. Interpolation is also used to simplify complicated functions by sampling data points and interpolating them using a simpler function [12].

Data is given only at discrete points such as $\left(\mathrm{x}_{0}, \mathrm{y}_{0}\right),\left(\mathrm{x}_{1}, \mathrm{y}_{1}\right), \ldots \ldots,\left(\mathrm{x}_{\mathrm{n}-1}, \mathrm{y}_{\mathrm{n}-1}\right)$, $\left(\mathrm{x}_{\mathrm{n}}, \mathrm{y}_{\mathrm{n}}\right)$.A continuous function $\mathrm{f}(\mathrm{x})$ may be used to represent the $\mathrm{n}+1$ data values 


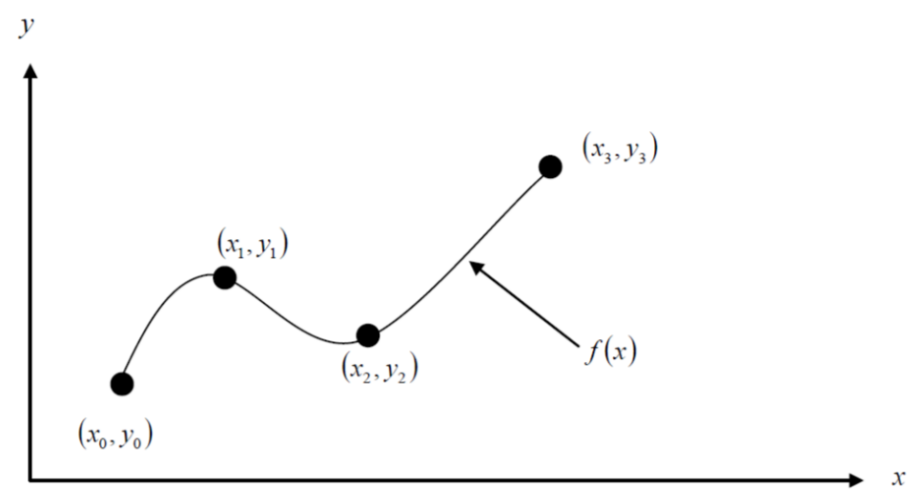

Figure 2.10: Interpolation Algorithm [16].

with $\mathrm{f}(\mathrm{x})$ passing through the $\mathrm{n}+1$ points (Figure ). Then one can find the value of $y$ at any other value of $x$. This is called interpolation. Of course, if $x$ falls outside the range of $\mathrm{x}$ for which the data is given, it is no longer interpolation but instead is called extrapolation. A polynomial is a common choice for an interpolating function because polynomials are easy to evaluate, differentiate, and Integrate relative to other choices such as a trigonometric and exponential series. Polynomial interpolation involves finding a polynomial of order $n$ that passes through the $n+1$ points. One of the methods of interpolation is called the direct method. Other methods include Newton's divided difference polynomial method and the Lagrangian interpolation method. 


\section{Chapter 3}

\section{Proposed Method}

In this chapter, we discussed in details which algorithms we actually used for classification, segmentation and 3D visualization of the tumor. Our proposed model is composed of three parts which are: classification with CNN, Segmentation and 3D visualization. 3.1 At first, we take an image input which is MRI Dicom image file. In the Convolution Neural Network model, we classify the images whether there is a tumor or not 3.2. In Segmentation, we divided the work into two phases such as Pre-processing and Processing. In pre-processing, we used artifacts removal function to get rid of visual artifacts from MRI images to have more realistic original image. Next, to remove more noise from the image we use several filters like Median, Average, Gaussian and Un-sharped Masking filter. Then, we use histogram normalization to detect the tumor affected MRI image. In the Processing part, we mainly use Otsu's multilevel thresholding algorithm to extract the interest region from the images (Reference the figure number). After processing, in 3D visualization stage where we get our 3D visualization of the brain tumor by using polynomial interpolation algorithm where synthetic layers are made to build surfaces.

\subsection{DataSet Preparation}

The paper uses the TCIA 2013 databases. There are four MRI sequences available for every patient: T1-weighted (T1), T1-weighted and contrast-enhanced (T1), T2weighted (T2) and T2-weighted FLAIR (FLAIR). All images in the TCIA 2013 databases have the same sequences. The training image data consists of different MRI slices Dicom image from patients and each image contains pixel size of $640 \mathrm{x}$ 640. As our main concern of interest is to find the tumor infected area of the brain for that reason we mainly focus on image pixel quality. To reduce the computational cost of the algorithm we have converted the image RGB to Grayscale.

\subsection{Implementation of Convolution Neural Net- work}

Since the brains in the TCIA dataset lack resolution in the third dimension, we consider performing the segmentation slice by slice from the axial view. Thus, our model processes sequentially each 2D axial image (slice) where each pixel is associated with different image modalities namely; T1, T2, T1C, and FLAIR. Like 


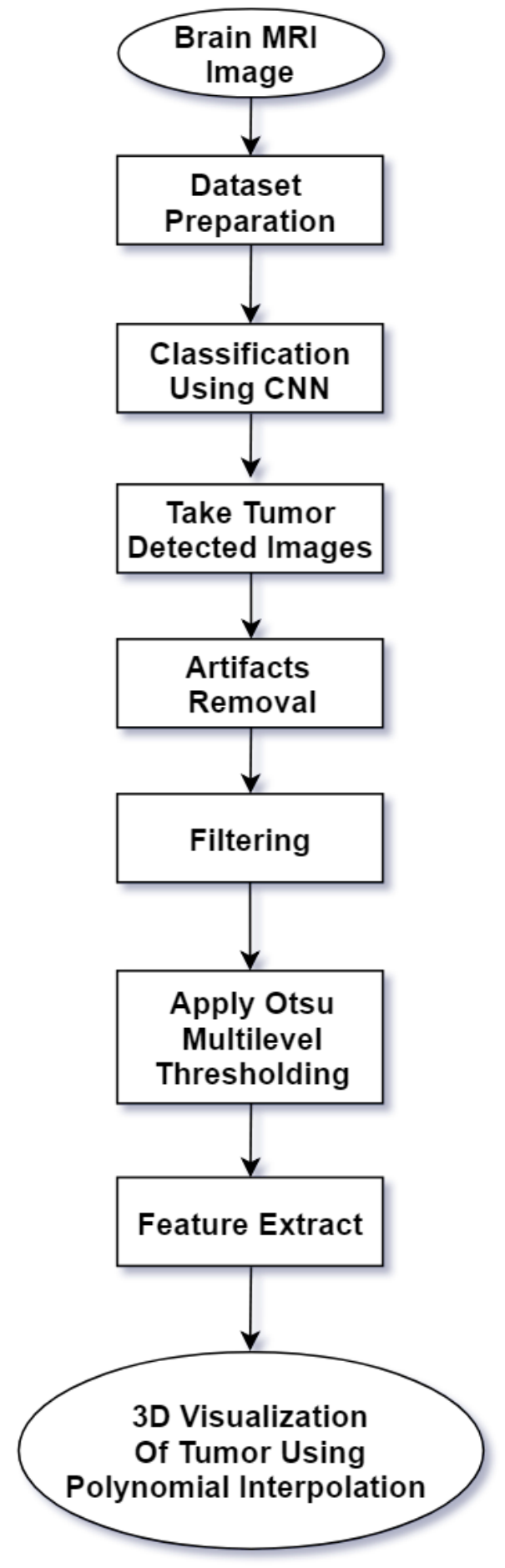

Figure 3.1: Work flow of Proposed Model. 


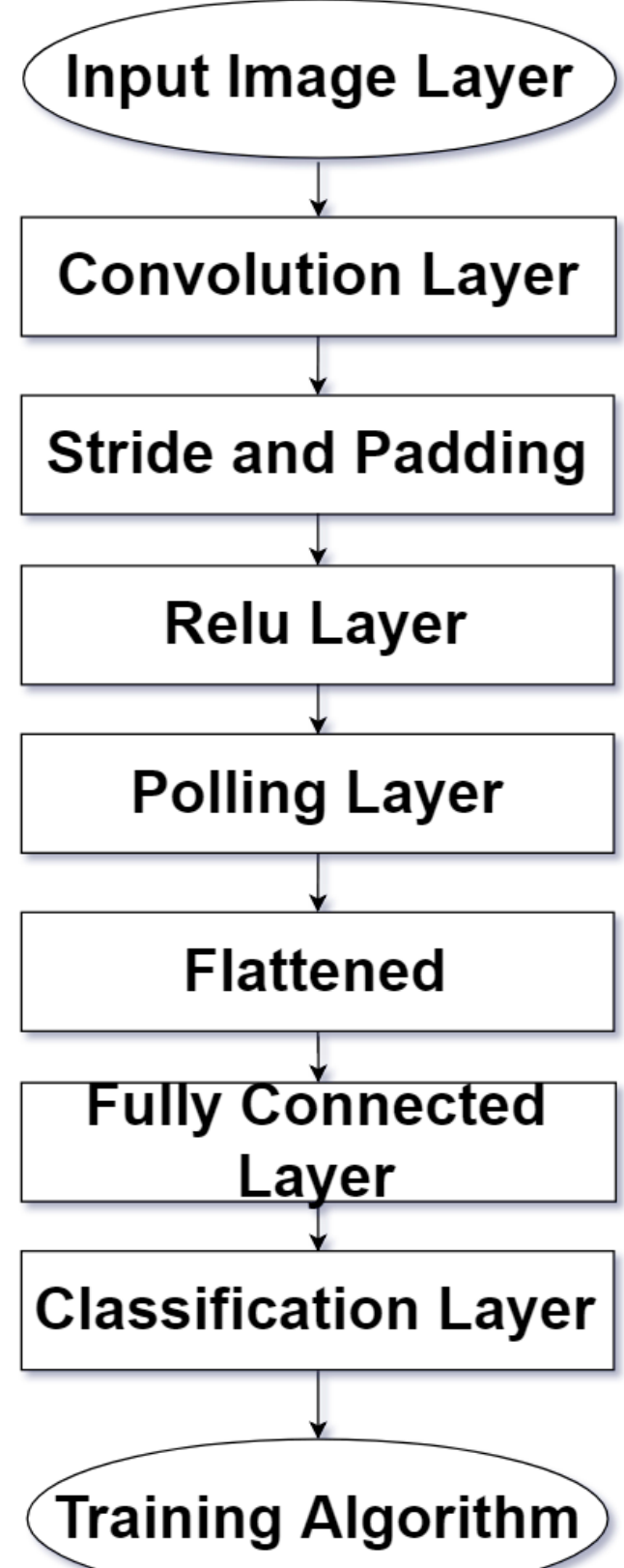

Figure 3.2: Implementation of Convolution Neural Network. 
most CNN-based segmentation models our method predicts the class of a pixel by processing the $\mathrm{M} \times \mathrm{M}$ patch centered on that pixel. The input $\mathrm{X}$ of our CNN model is thus an M x M 2D patch with several modalities. The main building block used to construct a CNN architecture is the convolutional layer. Several layers can be stacked on top of each other forming a hierarchy of features. Each layer can be understood as extracting features from its preceding layer into the hierarchy to which it is connected. A single convolutional layer takes as input a stack of input planes and produces as output some number of output planes or feature maps. Each feature map can be thought of as a topologically arranged map of responses of a particular spatially local nonlinear feature extractor (the parameters of which are learned), applied identically to each spatial neighborhood of the input planes in a sliding window fashion. In the case of a first convolutional layer, the individual input planes correspond to different MRI modalities (in typical computer vision applications, the individual input planes correspond to the red, green and blue color channels). In subsequent layers, the input planes typically consist of the feature maps of the previous layer.

\subsubsection{Input Image Layer}

This is the first layer of the convolutional neural network. Input data number depends on image size and dimension. If an image is $200^{*} 150^{*} 3$ then the input data number will be 90,000 for a single image.For our dataset, we have MRI images which generally build with DICOM format.To reduce the computational we have converted that image files to RGB to Grayscale. As our main concern of interest is to find the infected area of the brain which is caused by the tumor. Our dataset image content $640^{*} 640$ pixel size in grayscale value and has different slices.

\subsubsection{Convolution Layer}

The second layer of a convolutional neural network is a 2D convolution layer. In this layer, all the input image is convolved with a filter matrix and output of convolution layer is called a feature map or activation map. If the image is expressed by $\mathrm{I}(\mathrm{x}$, $\mathrm{y})$, the filter is expressed by $\mathrm{F}(\mathrm{x}, \mathrm{y})$, invert then shifting of the filter is $\mathrm{F}(-\mathrm{u}+\mathrm{x}$, $-\mathrm{v}+\mathrm{y})$. The output of the convolution layer is a matrix that represents where an identical feature is active in the input matrix indicating filter activation. The output matrix number (feature number) will be the same as the number of filters taken for convolution. The output of the convolution layer is a matrix that represents where an identical feature is active in the input matrix indicating filter activation. The output matrix number (feature number) will be the same as the number of filters taken for convolution. In our work, we have reshaped our input image by $100 * 100$ pixel size to reduce the cost by using an image processing library (such as CV2). We also make image size equal to minimize the cost.Filter also called kernel which is a matrix that represents feature such as curve, edge, shape, etc. Bigger filter size represents a bigger shape but it can convolve in lower number with the input image. Therefore, the convolution output will be lower in size. The different filter produces different feature map. Increased number of filters will produce a larger number of the feature map. The depth of filter depends on the depth of the matrix to be convolved with a filter. The first required Conv2D parameter is the number of filters that the 
convolutional layer. The exact range of the values may be different for you, but start with a smaller number of filters and only increase when necessary.In our work, we have set the filter size to 256 because depending on the complexity of our dataset. The second required parameter you need to provide to the Keras Conv2D class is the kernel_size, a 2-tuple specifying the width and height of the $2 \mathrm{D}$ convolution window.Depending on the pixel size we have set kernel size is equal to $3 \times 3$ matrix as our image pixel size is not greater than 128 .

\subsubsection{Stride and Padding}

Stride is the number of pixels shifts over the input matrix. The strides parameter is a 2-tuple of integers, specifying the "step" of the convolution along the $\mathrm{x}$ and $y$-axis of the input volume When the stride is 1 then we move the filters to 1 pixel at a time. The strides value defaults to $(1,1)$, implying that: A given convolutional filter is applied to the current location of the input volume. The filter takes a 1-pixel step to the right and again the filter is applied to the input volume. This process is performed until we reach the far-right border of the volume in which we move our filter one pixel down and then start again from the far left. In our project, we have set the stride $=1$ to get the maximum value from the images. Sometimes filter does not fit perfectly fit the input image. The padding parameter to the Keras Conv2D class can take on one of two values: valid or same. With the valid parameter, the input volume is not zero-padded and the spatial dimensions are allowed to reduce via the natural application of convolution.If we instead want to preserve the spatial dimensions of the volume such that the output volume size matches the input volume size, then you would want to supply a value of same for the padding. In our case, we have used to valid padding to extract the spatial dimension on the image.

\subsubsection{Relu Layer}

To obtain features that are non-linear transformations of the input, an elementwise non-linearity is applied to the result of the kernel convolution. There are multiple choices for this non-linearity, such as the sigmoid, hyperbolic tangent and rectified linear functions. If a filter has negative pixel value then the convolution output may be negative. Negative value has no significance without representing the lowest activation in the image. Therefore, we need to rectify these values. This layer provides non-linear output to the next layer. Researcher normally uses the sigmoid and tanh function for non-linear output but the rectification process is better because of its computational efficiency. The activation parameter to the Conv2D class is simply a convenience parameter, allowing you to supply a string specifying the name of the activation function you want to apply after performing the convolution. We have used to sigmoid as the activation parameter of Conv2D.

\subsubsection{Pooling layer}

The Pooling layer can be seen between Convolution layers in a CNN architecture. This layer basically reduces the number of parameters and computation in the network, controlling overfitting by progressively reducing the spatial size of the network. There are two operations in this layer; Average pooling and Maximum pooling. Maxpooling, like the name states; will take out only the maximum from a pool. This is 
actually done with the use of filters sliding through the input; and at every stride, the maximum parameter is taken out and the rest is dropped. This actually downsamples the network.In our project, we have used Max-pooling as we need the pixel value which is high because it contains much of the information.Our input image is grayscale so if we apply max -polling we get the maximum value that is every image contains. The pooling size of our project is $2 * 2$.

\subsubsection{Flattened}

The last stage of a convolutional neural network (CNN) is a classifier. It is called a dense layer, which is just an artificial neural network (ANN) classifier. And an ANN classifier needs individual features, just like any other classifier. This means it needs a feature vector. The flattening step is needed so that you can make use of fully connected layers after some convolutional layers. Fully connected layers don't have a local limitation like convolutional layers (which only observe some local part of an image by using convolutional filters). This means you can combine all the found local features of the previous convolutional layers. Each feature map channel in the output of a CNN layer is a "flattened" 2D array created by adding the results of multiple 2D kernels.

\subsubsection{Fully Connected Layer}

In this layer, the neurons have a complete connection to all the activations from the previous layers. Their activations can hence be computed with a matrix multiplication followed by a bias offset. This is the last phase for a CNN network. The Convolutional Neural Network is actually made up of hidden layers and fullyconnected layer(s). Output data of polling layer is a 2D cascade matrix. A number of $2 \mathrm{D}$ matrix is equivalent to a convolutional layer filter number. 2D matrices transfer to the $1 \mathrm{D}$ matrix in this layer then feed to the classification layer.

\subsubsection{Classification Layer}

A classification layer computes the cross entropy loss for multi-class classification problems with mutually exclusive classes. For typical classification networks, the classification layer must follow the softmax layer. This layer is the last layer of CNN that classify images depends on the information collected from a fully connected layer. Anyone needs to declare a class number for classification.

\subsubsection{Training Algorithm}

CNN train and update its weights and biases in different layer by Stochastic Gradient Descent with Momentum that is back propagation algorithm using train option parameter's information. In our project we have set (batch_size $=32$, epochs $=10$, validation_split $=0.3)$. That means it will train our data 10 times and $70 \%$ of our data for training and $30 \%$ of our data for testing. 


\subsection{Artifacts Remove}

In our case we considered visual artifacts which are anomalies appears during visual representation as in digital image files particularly microscopy, magnetic resonance imaging etc. We are considering digital artifacts, noise, distortion, image quality factors and compression artifacts [23]. We use strel function of MATLAB to represents a flat morphological structuring elements, which is essential part of morphological dilation and erosion operations. Strel function create flat structures elements with both binary and grayscale images. Figure 3.3, illustrates structuring element.

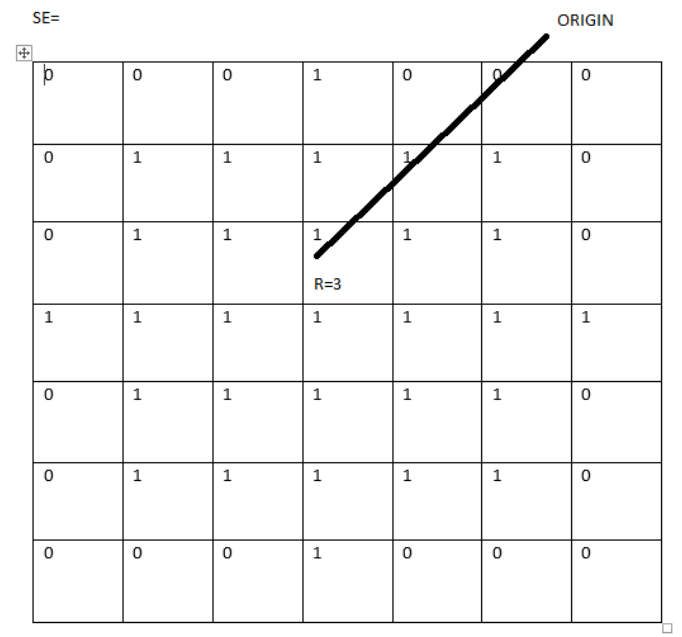

Figure 3.3: Structuring Element.

\subsection{Noise Remove}

Noise removal process is used to resolve distortion issues. In our work mainly we use median, average, gaussian filter and un-sharped musking filter to remove the noise of images.

\subsubsection{Median Filter}

Median filter is a nonlinear and digital filter. It removes the unwanted signals or noise from the input image. To be more specific, it takes the value of the pixels from the neighborhood because it should take in order from low to high. Finally, the calculation is considered as median. Moreover, it works perfectly against impulse noises.

$$
f(x, y)=\operatorname{median}(g(s, t))
$$

Here, $(s, t) \in S_{x y}$

The first estimation of the pixel is incorporated into the calculation of the median. 
Median filters are very well known in light of the fact that, for particular sorts of random noise they give fantastic noise reduction capabilities, with significantly less obscuring than linear smoothing filters of similar size [19]. The median is much less sensitive than the mean to extreme values(called outliers). Therefore, median filter is better able to remove these outliers without reducing the sharpness of image.

\subsubsection{Average Filter}

Average filter basically used for removing salt and pepper noise from an image and where we set the value of the output pixel to average of the pixel values in the neighborhood around the corresponding input pixels [21].

\subsubsection{Gauss Filter}

The Gaussian filter helps to blur the image. Moreover, it is also called a smoothing operator. It removes the fine details which are present in the image. It mainly defines the probability distribution of the noise [26]. To be more emphasis, it is very effective for the removal of the noise.

Gaussian function in 2D,

$$
G(x, y)=\frac{1}{2 \pi \sigma^{2}} e^{-\frac{x^{2}+y^{2}}{2 \sigma^{2}}}
$$

Here, $\sigma$ is acting as standard deviation. The intensity of smoothing is controlled by $\sigma$.

\subsubsection{Un-sharped Masking Filter}

Unsharp filter is an operator used to sharpen an image. By applying the algorithm, it helps the image clearer and it is an accurate representation of the image's subject. Unsharp masking is a linear image processing technique [6]. The blurring filter step could use any image filter.

\subsection{Comparison Parameter}

To compare the performance of different filters, PSNR and MSE are used.

\section{Mean Square Error}

MSE measures the average of square of the errors which is the average squared difference between the estimated values and what is estimated. Basically MSE is nothing but our loss function. Lesser MSE is more better the estimator. In the previous equation, $\mathrm{M}$ and $\mathrm{N}$ are the number of rows and columns in the input images, respectively. Then the block computes the PSNR using the following equation:

$$
M S E=\sum_{M, N}\left[I_{1}(m, n)-I_{2}(m, n)\right]^{2}
$$

Here, $I_{1}(m, n)$ is original $I_{2}(m, n)$ image and is denoised image [25]. 


\section{Peak Signal to Noise Ratio}

PSNR block computes the peak signal to noise ratio, in decibels between two images. This ratio is mainly used as quality measurement between original and compressed image. The higher PSNR is more better quality of compressed. To compute the PSNR, the block first calculates the mean-squared error using the following equation:

$$
P S N R=10 \log _{10}\left(\frac{R^{2}}{M S E}\right)
$$

Here, $\mathrm{R}$ is defined as maximum value of pixel present in an image and MSE is the mean square error between original and denoised image with $\mathrm{M}^{*} \mathrm{~N}$ size [25].

\subsubsection{Segmentation}

OTSU's clustering-based image thresholding algorithm is used to segment the tumor of MRI image.

\section{OTSU's Multilevel Thresholding Algorithm}

OTSU's algorithm is named after its inventor Nobuyuki Otsu. This is one of the better threshold methods for real world images to different shape mesurement. This methods works better for complex background [15]. In this algorithm, there are two types of pixel such as foreground and background. The process of multi-level thresholding can be devided into five steps [15],

1. Step 1: Image acquisition is used to eliminate noise from image.

2. Step 2: Gradient is calculation along the $\mathrm{x}$ and $\mathrm{y}$ axis.

3. Step 3: Value of gradient is used to separate two different threshold.

4. Step 4: At the forth stage, different threshold level 3 and level 4 are calculated.

5. Step 5: Edge of image will be generated.

Flow chart for edge-detection method is shown in 3.4 .

An image is a 2D grayscale intensity function, and contains $\mathrm{N}$ pixels with gray levels from 1 to $\mathrm{L}$ [8]. The number of pixels with gray level $\mathrm{i}$ is denoted $f_{0}$, giving a probability of gray level $i$ in an image of

$$
p_{i}=f_{i} / N
$$

In the case of bi-level thresholding of an image, the pixels are divided into two classes, $C_{1}$ with gray levels $[1, \ldots, \mathrm{t}]$ and $C_{2}$ with gray levels $[\mathrm{t}+1, \ldots, \mathrm{L}]$. Then, the gray level probability distributions for the two classes are

$$
C_{1}: p_{1} / \omega_{1}(t), \ldots p_{t} / \omega_{1}(t)
$$

and

$$
C_{2}: p_{t+1} / \omega_{2}(t), p_{t+2} / \omega_{2}(t), \ldots p_{L} / \omega_{2}(t)
$$




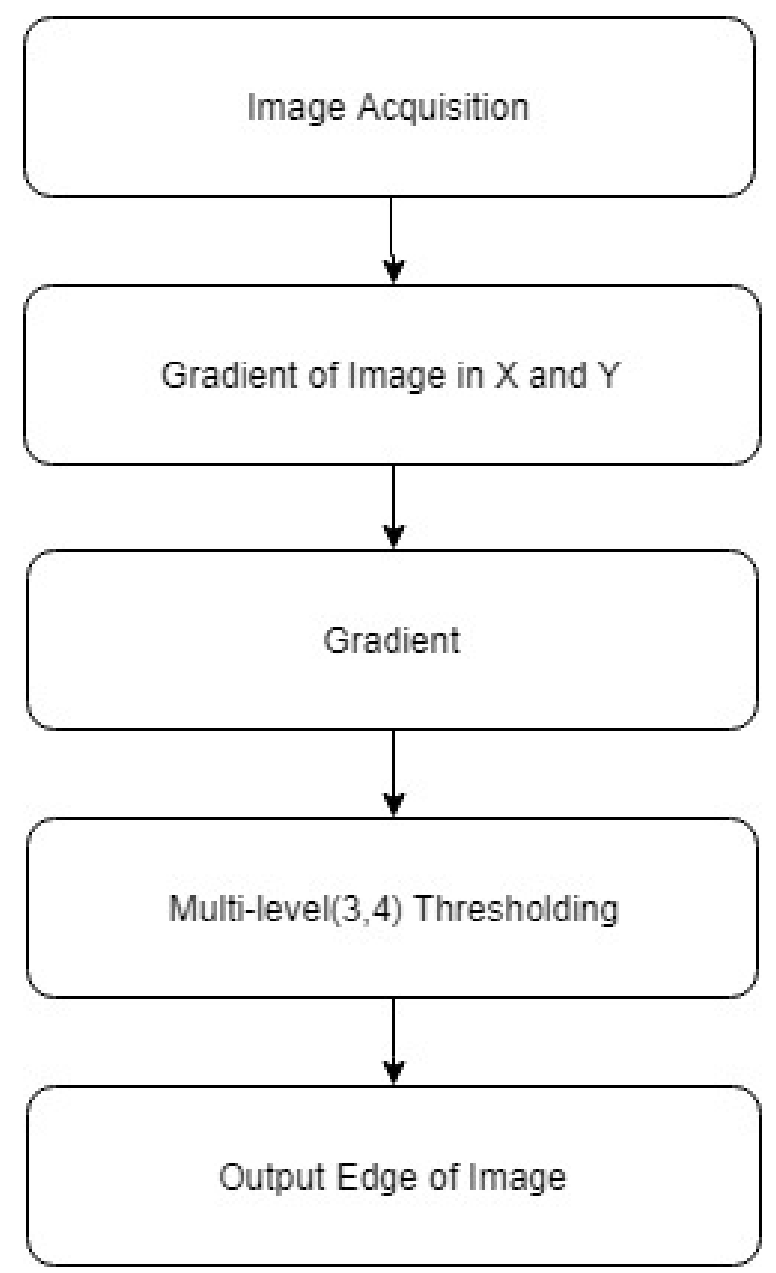

Figure 3.4: Process diagram of edge-detection by multi-level thresholding. 
where

$$
\omega_{1}(t)=\sum_{i=1}^{L} p_{i}
$$

and

$$
\omega_{2}(t)=\sum_{i=t+1}^{L} p_{i}
$$

Also, the means for classes $C_{1}$ and $C_{2}$ are

$$
\mu_{1}=\sum_{i=1}^{t} i p_{i} / \omega_{1}(t)
$$

and

$$
\mu_{2}=\sum_{i=t+1}^{L} i p_{i} / \omega_{2}(t)
$$

Let $\mu_{T}$ be the mean intensity for the whole image. It is easy to show that

$$
\begin{gathered}
\omega_{1} \mu_{1}+\omega_{2} \mu_{2}=\mu_{T} \\
\omega_{1}+\omega_{2}=1
\end{gathered}
$$

Using discriminant analysis, Otsu defined the between-class variance of the thresholded image as [8]

$$
\omega_{B}^{2}=\omega_{1}\left(\mu_{1}-\mu_{T}\right)^{2}+\omega_{2}\left(\mu_{2}-\mu_{T}\right)^{2}
$$

In Matlab, we used the built-in function multithresh() which is implemented with Otsu's Multilevel Thresholding algorithm. 


\section{Chapter 4}

\section{Experiments and Results}

In this chapter, we will try to illustrate the experimental results of our proposed model.

\subsection{Accuracy Measurement}

We have used 800 MRI images to measure the accuracy of our proposed model algorithm. Where we had 422 tumor affected and 378 non tumor affected images. Among them, we got positive result of 362 as TP and 320 as TN respectively. On the other side, we had got 58 as $\mathrm{FP}$ and 60 as $\mathrm{FN}$ respectively.

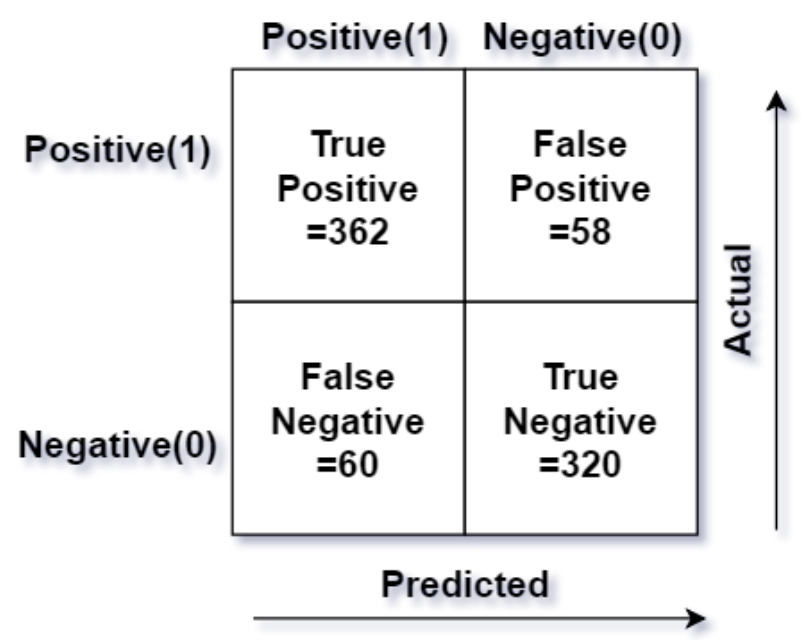

Figure 4.1: Confusion Matrix.

We can show the result into true positive (TP), true negative (TN), false positive $(\mathrm{FP})$ and false negative $(\mathrm{FN})$ regions. TP represents pixels where tumor was detected and it should be tumor. TN means that tumor was not detected and should not be. FP is when tumor was detected and should not be. Finally if tumor was not detected, but should be, it is FN [6].

Our Experimental,

True positive $=362$

True negative $=320$ 
False positive $=58$

False negative $=60$

So, the evaluation results will be represented by the following equations,

$$
\begin{gathered}
\text { Accuracy }=\frac{(T P+T N)}{(T P+T N+F P+F N)} \\
=\frac{(362+320)}{(362+320+58+60)} \\
=\frac{682}{800} \\
=0.8525
\end{gathered}
$$

Finally, the accuracy of our proposed system is $85.25 \%$

\subsection{Image Pre-processing}

In our proposed system image pre-processing is divided into two parts.

1. Artifacts Remove

2. Noise Remove

\subsubsection{Artifacts Remove}

Before applying our proposed image processing techniques, we have to remove the artifacts from the images by using morphological open operation.

Function strel is used for applying morphological structuring element where se $=$ strel (shape, radius) is the syntax of that function. Here, shape is a string which defines different type of shapes such as disk, diamond, square etc and radius is a integer which defines different pixels value. To remove artifacts from the images, we used disk shape and 1 to 5 pixel value is taken as parameter input and measured the performance of the image. 

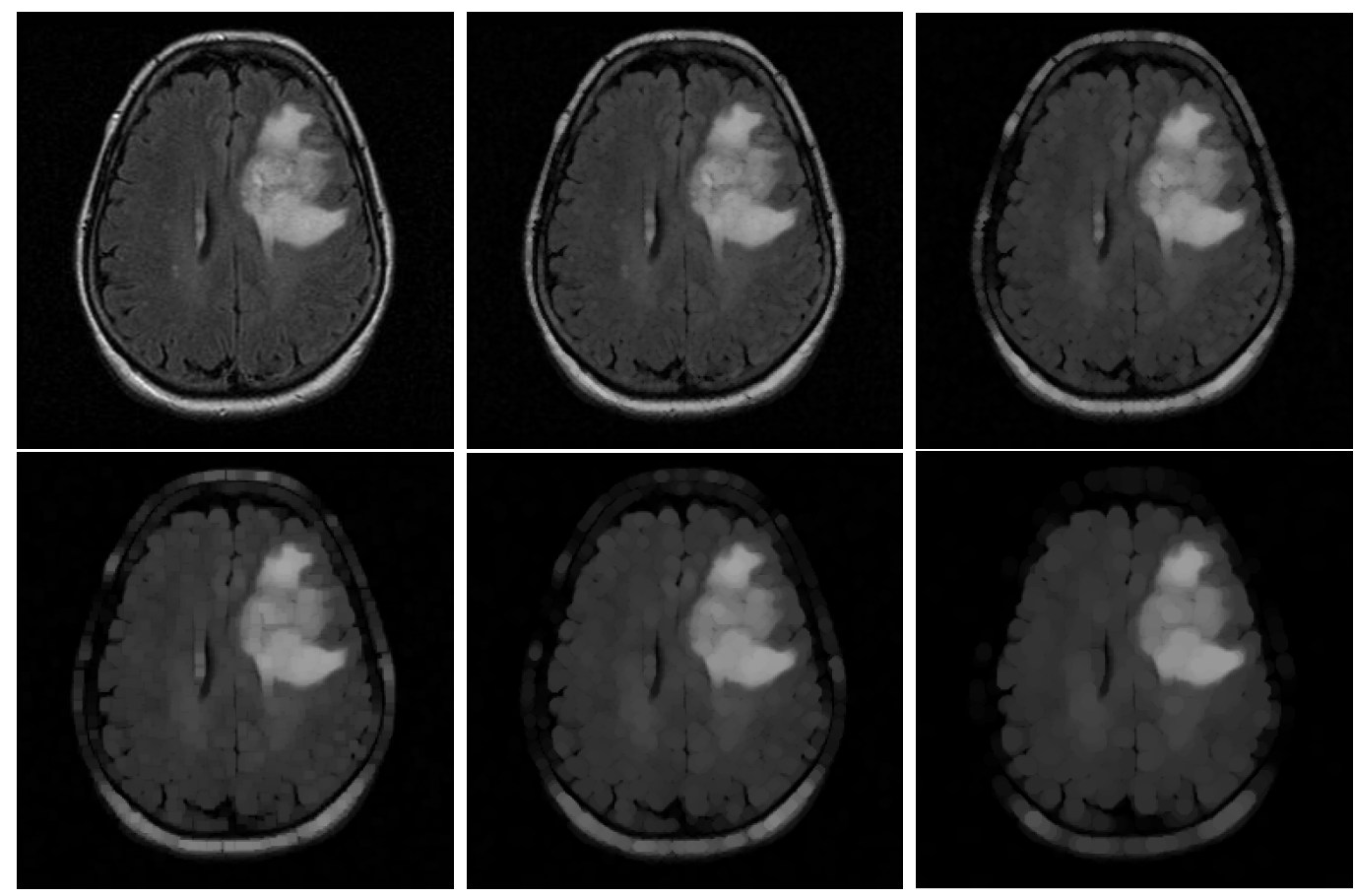

Figure 4.2: Artifacts removal results using different pixel value.

In Figure 4.2 (a) Original MRI image of jpeg form, (b) Morphological operation based on 1 pixel disk radius, (c) Morphological operation based on 2 pixels disk radius, (d) Morphological operation based on 3 pixels disk radius, (e) Morphological operation based on 5 pixels disk radius. Explanation of Figure 4.2,

a) This is the original image by which we performed artifacts removal operation.

b) For 1 pixels we get satisfactory result. Many artifacts are removed from the original image.

c) 2 pixels disk radius give more better results but some of artifacts are still found in the image.

d) 3 pixels disk radius give best result than all others and image quality is also good.

e) For 4 pixels disk radius, result is not satisfactory and image is not clear.

f) 5 pixels disk radius gives the worst result among all of them.

For our dataset, 3 pixel disk radius gives the best and satisfactory result. We have used 3 pixels radius artifacts removed image for applying further algorithms.

\subsubsection{Noise Remove}

To remove the noise of an image, we have applied several image filtering algorithms such as,

a) Median Filter 


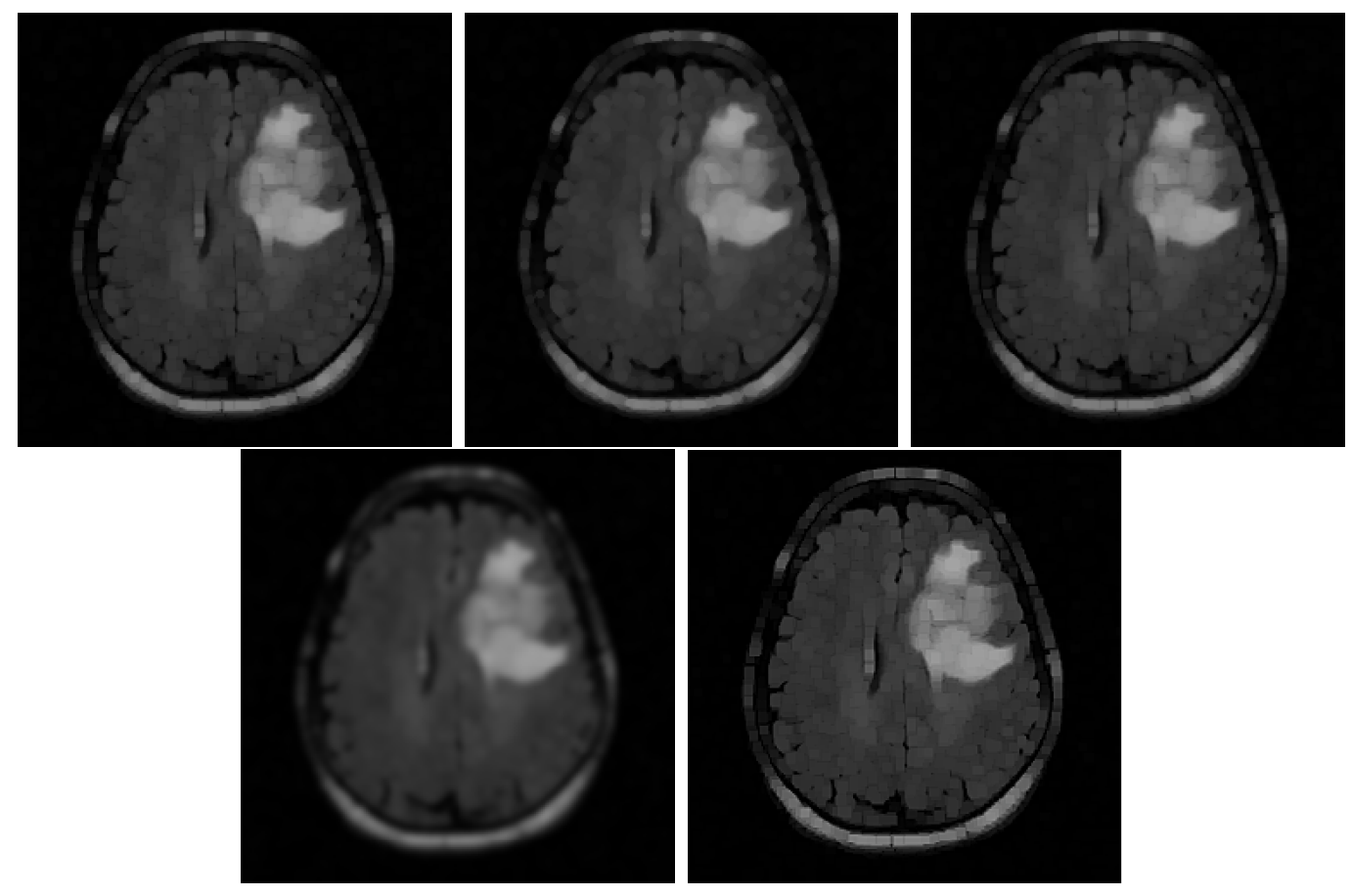

Figure 4.3: Noise removal results using different filters.

b) Average Filter

c) Gaussian Filter

d) Un-sharped Masking Filter

Explanation of Figure 4.3,

a) Original MRI image after applying 3 pixels disk radius morphological opening operation.

b) Median filter has done best among all of the filters. Noise has been removed properly and edges of the image also clear.

c) Average filter also gives the better result. This case edges of the image is not clear also.

d) For gaussian filter, image has become much blur also reduce the quality of image.

e) Un-sharped masking filter has performed well. Noise of the image is removed and image quality is good but edge is not clear.

To measure the performance of these filtering approach, we used Peak Signal-toNoise Ratio (PSNR) and Mean-Squared Error (MSE) to measure of image quality. The table describes average of $124 \mathrm{~T} 1, \mathrm{~T} 2$ and Flair images.

For our dataset, Mean and un-sharped masking filtering give the more better results than other filtering results. Among mean and un-sharped masking filtering, unsharped masking filtering gives little better result than median filter. We have used un-sharped masking filtering image for applying further algorithms. 
T1 Image

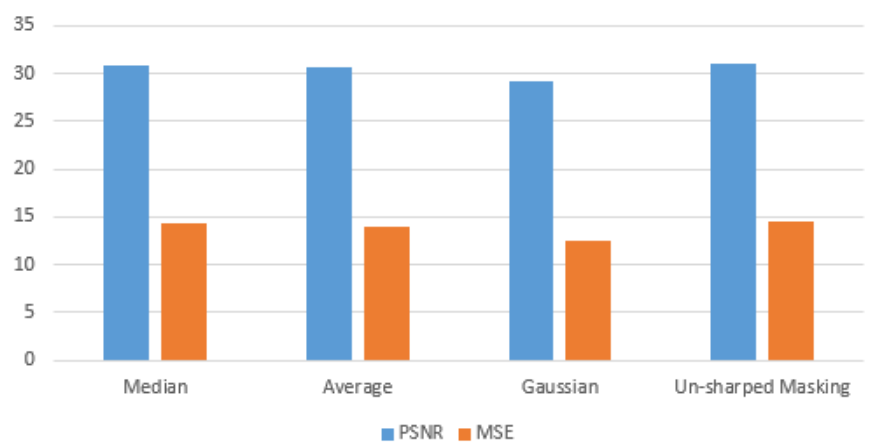

Figure 4.4: Overview of the result of PSNR and MSE on T1.

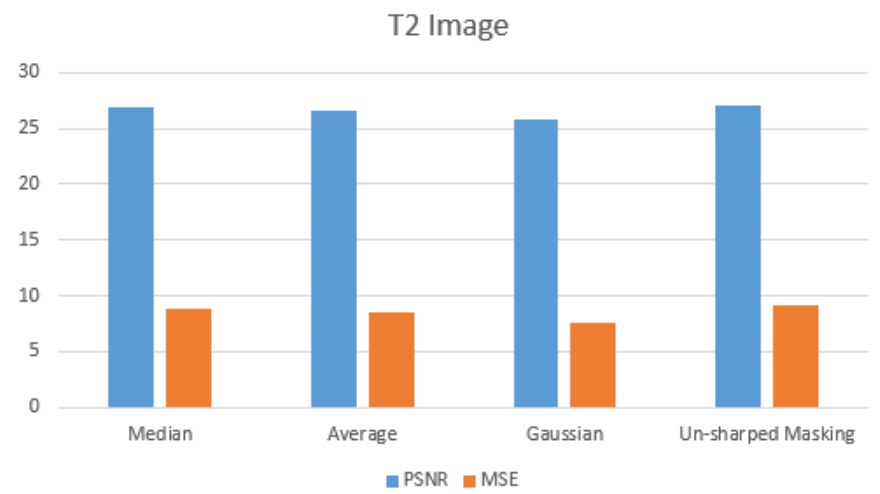

Figure 4.5: Overview of the result of PSNR and MSE on T2.

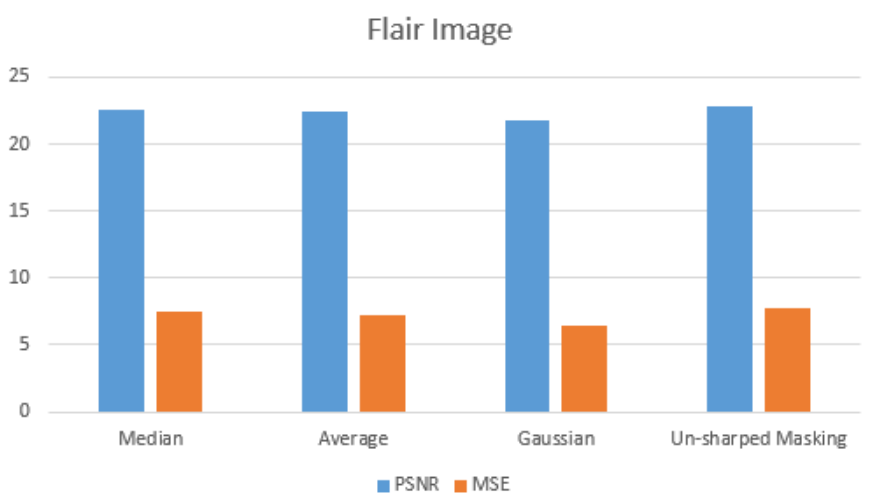

Figure 4.6: Overview of the result of PSNR and MSE on Flair. 
Table 4.1: The average PSNR and MSE results

$2 *$ Method

Median Filter

Average Filter

Gaussian Filter

Un-sharped Masking Filter

\begin{tabular}{cccccc}
\multicolumn{3}{c}{ PSNR } & \multicolumn{3}{c}{ MSE } \\
\hline T1 & T2 & Flair & T1 & T2 & Flair \\
30.8669 & 26.8527 & 22.6270 & 14.2521 & 8.8314 & 7.5181 \\
30.6391 & 26.6582 & 22.46215 & 13.9821 & 8.5869 & 7.2706 \\
29.2575 & 25.7859 & 21.7453 & 12.4869 & 7.6166 & 6.3907 \\
31.0105 & 27.0188 & 22.791 & 14.455 & 9.0826 & 7.7950
\end{tabular}

\subsubsection{Tumor Segmentation}

In our proposed model, we tried to identify not only the affected tumor area but also separating more and less affected area in brain. For this purpose, multilevel image thresholds using Otsu's method has been used. Moreover, Otsu's method is comparatively faster than other multilevel image thresholds [5].

Functions we used to apply Otsu's multilevel image thresholds are,

$$
\begin{gathered}
\text { thresh }=\text { multithresh }(A, 3) ; \\
\text { seg }=\text { imquantize }(A, \text { thresh }) ; \\
r g b=\text { label } 2 r g b(\text { seg }) ;
\end{gathered}
$$

Function multithresh is used for applying Otsu multilevel image threshold where thresh $=$ multithresh $(\mathrm{A}, \mathrm{N})$ is the syntax of the function. Here, $\mathrm{A}$ is the input gray image by which we want to apply Otsu's algorithm and $\mathrm{N}$ represents threshold values that means how many levels we want. Value of $\mathrm{N}$ is 3 , where all the sections like identifying the normal brain, less affected area and more affected area will get 1 as their value respectively.

Function imquantize is used for segment the image into levels where seg = imquantize $(\mathrm{A}$, thresh) is the syntax of that function. Here, $\mathrm{A}$ is the input gray image and levels contains $\mathrm{N}+1$ discrete integer values in the range 1 to $\mathrm{N}+1$. That means, the levels of our proposed model will be 4 .

Function label2rgb converts label matrix into rgb image where rgb = label2rgb(L) is the syntax of that function.

Explanation of Figure 4.7,

(a) First Original tumor affected MRI image after applying proposed image pre-processing

(b) Segmentation of the MRI image of (a). Here red and yellow is the tumor affected area.

(c) Another Original tumor affected MRI image after applying proposed image pre-processing

(d) Segmentation of the MRI image of (c). Here red and yellow is the tumor affected area. 


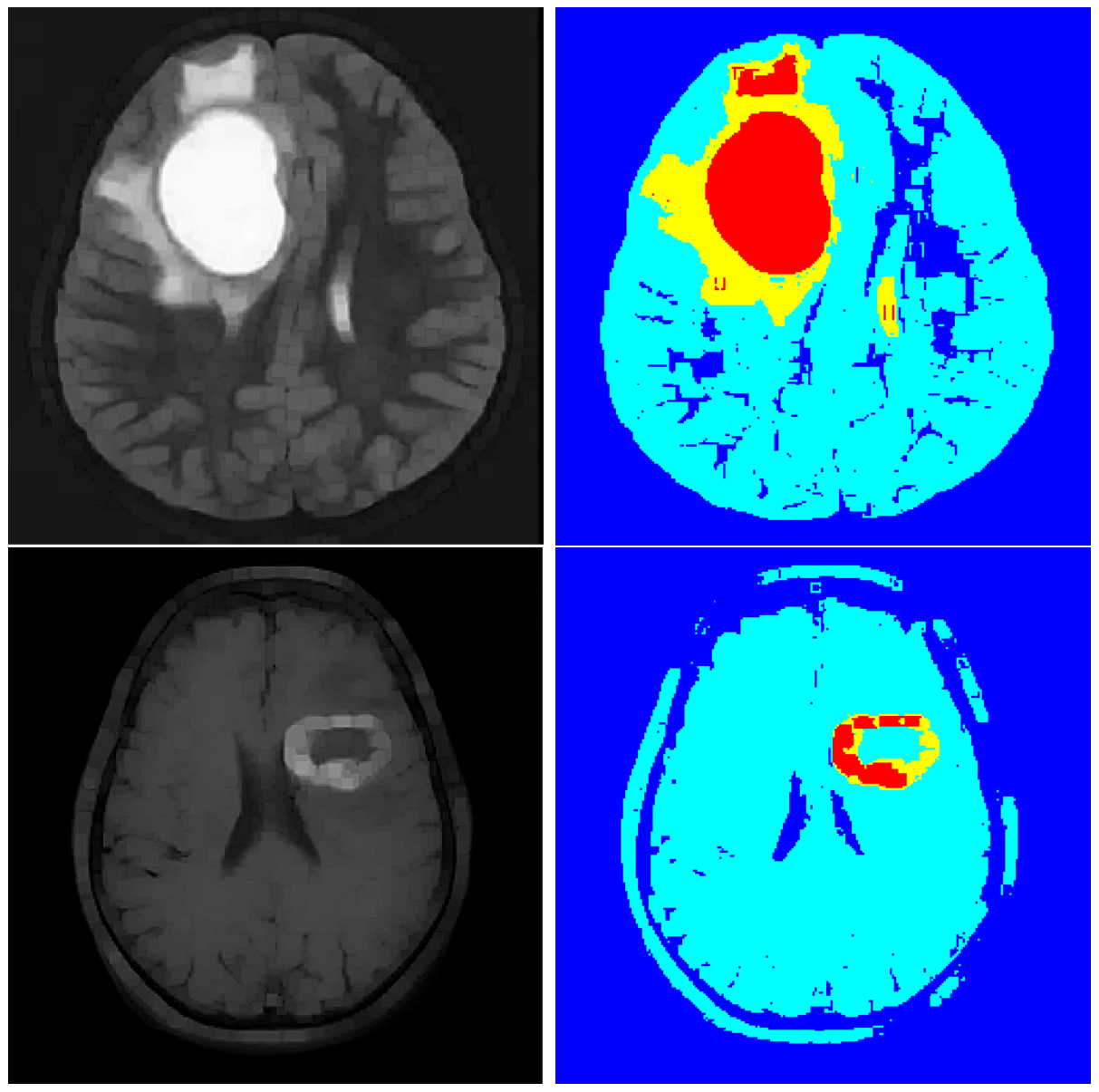

Figure 4.7: Image Segmentation. 


\subsubsection{D Visualization}

In $3 \mathrm{D}$ visualization part, we extract brain skull from our image and focus on tumor section.

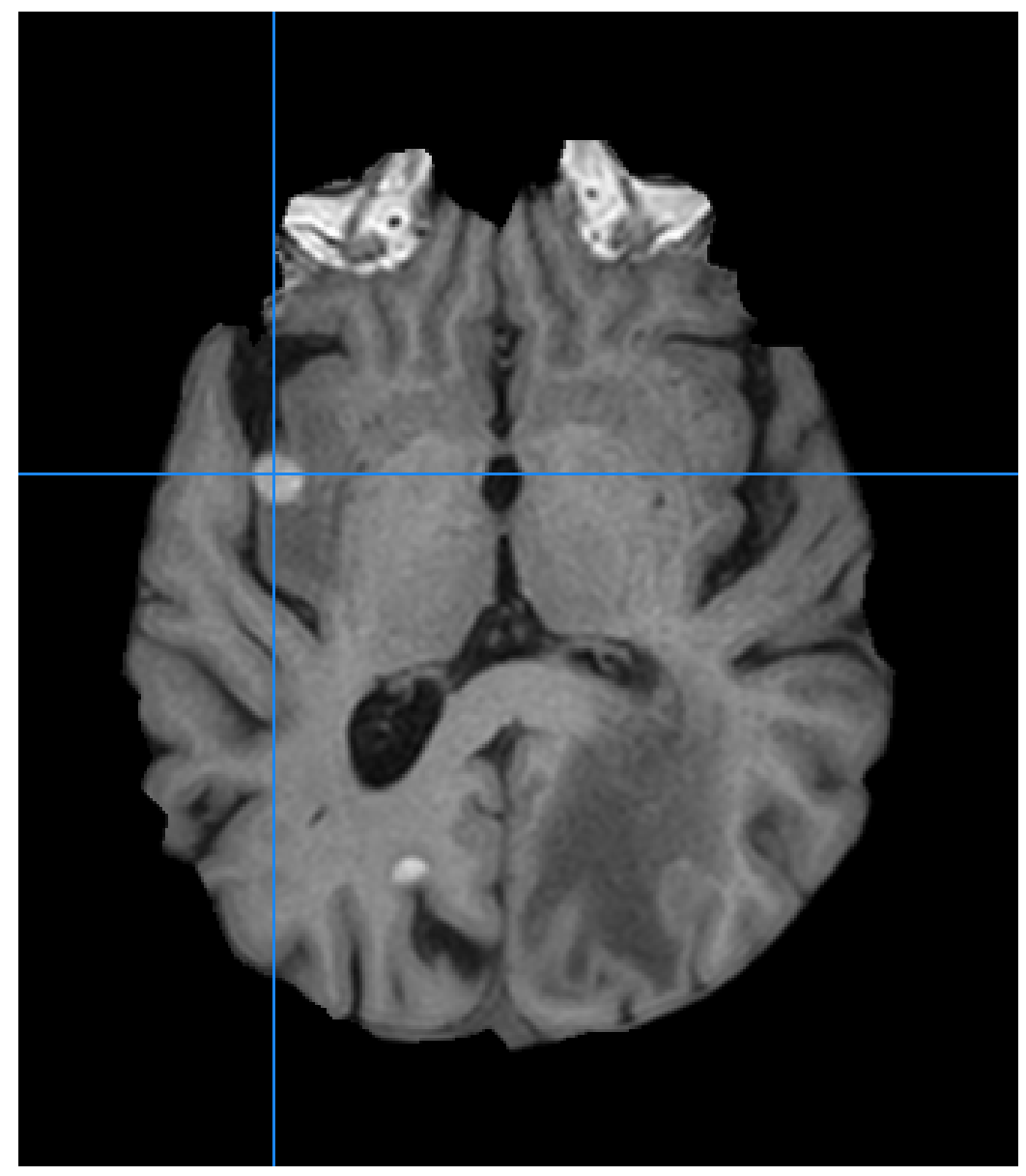

Figure 4.8: Axial View of Tumor.

To create 3D visualization of tumor, we use polynomial interpolation which will create new synthetic layers by constructing new data point within the range of closest known pixels. 

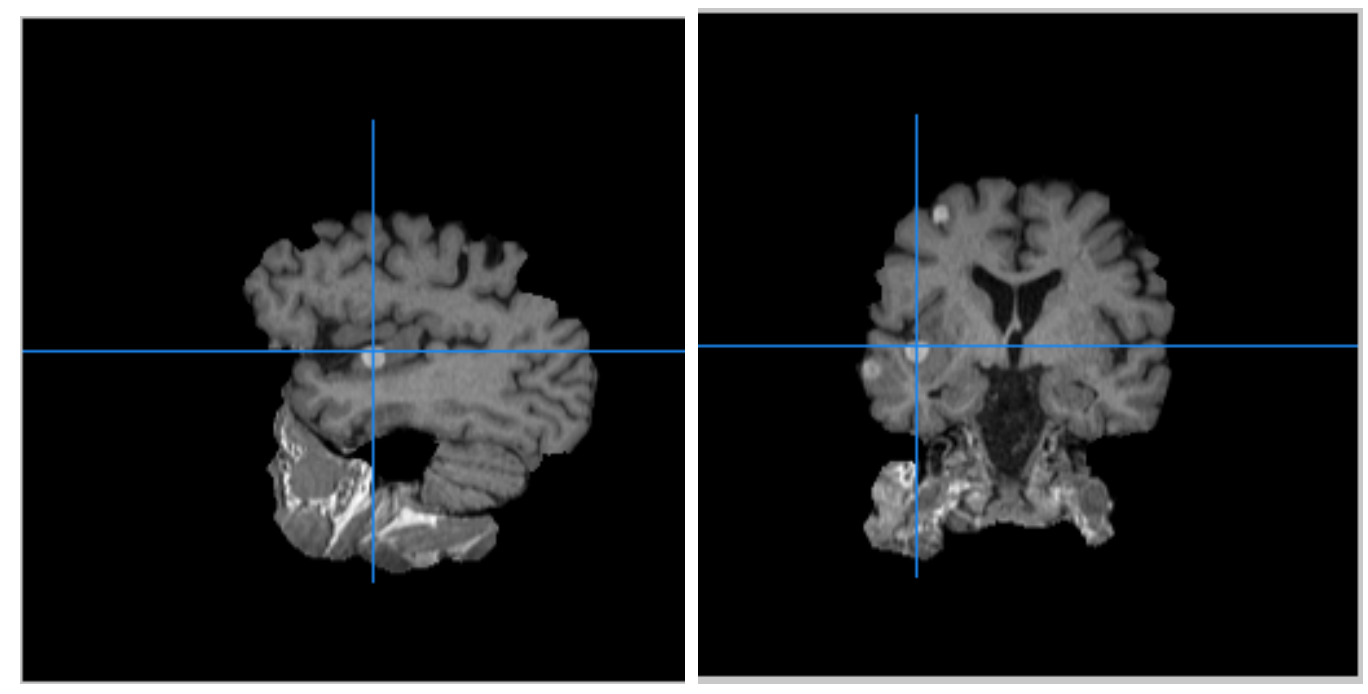

Figure 4.9: Segital Section (on Left) Cornal Section (on Right).
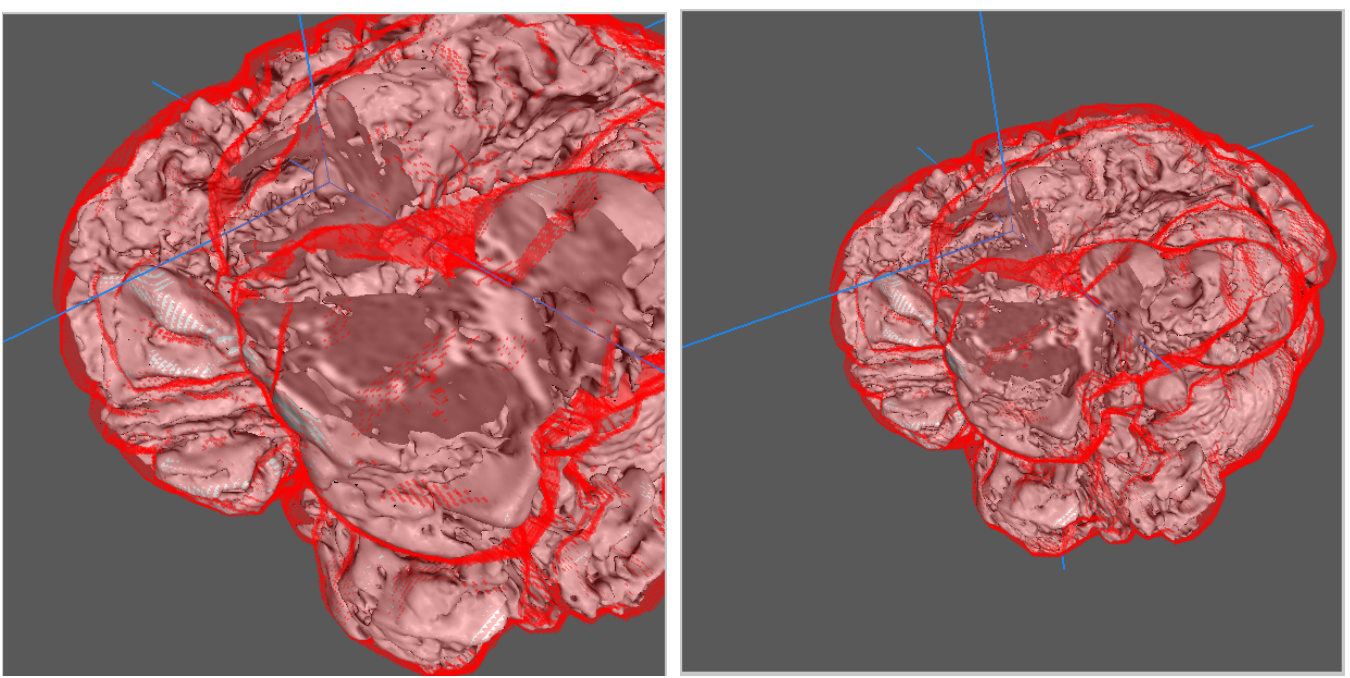

Figure 4.10: 3D Visualization of Identified Tumor. 


\section{Chapter 5}

\section{Conclusion and Future Works}

\subsection{Concluding Remark}

From the experimental results using CNN algorithm, all the methods were selected by using the trial-and-error technique. Different parameters were tuned on each progression and the best parameters were chosen based on classification accuracy. At first, We used Convolutional Neural Networks which is widely used for image classification tasks. In CNN, MRI brain tumor images are trained from scratch and we get $85 \%$ accuracy on that model. Next, we use basic image processing techniques notably OTSU's multi thresholding segmentation for extracting region of interest portion from image. Later, Based on extracted portion we use polynomial interpolation to create $3 \mathrm{~d}$ visualization of detected tumor. At conclusion, we would like to represent that our whole purpose of this research paper is to detect tumor from MRI images and visualize detected tumor in 3D more accurately compare to existing model. The proposed method achieved optimistic result which can be further improved and worked on in the near future.

\subsection{Future Works}

Future work includes the extension of this proposed approach for detecting different types of tumor in all parts of the human body and to find the actual size of the tumor. In this project, we were only able to visualize the tumor in 3D. We used raw sequential CNN model for thesis purpose. However for the future work pre-trained open source CNN model can be used for faster implementation and better accuracy through further hyper parameter tuning. 


\section{Bibliography}

[1] S. G. Mallat, "A theory for multiresolution signal decomposition: The wavelet representation", IEEE transactions on pattern analysis and machine intelligence, vol. 11, no. 7, pp. 674-693, 1989.

[2] K. K. Kwong, J. W. Belliveau, D. A. Chesler, I. E. Goldberg, R. M. Weisskoff, B. P. Poncelet, D. N. Kennedy, B. E. Hoppel, M. S. Cohen, and R. Turner, "Dynamic magnetic resonance imaging of human brain activity during primary sensory stimulation.", Proceedings of the National Academy of Sciences, vol. 89, no. 12, pp. 5675-5679, 1992.

[3] V. Chandnani, T. Yeager, T. DeBerardino, K. Christensen, J. Gagliardi, D. Heitz, D. Baird, and M. Hansen, "Glenoid labral tears: Prospective evaluation with mri imaging, mr arthrography, and ct arthrography.", AJR. American journal of roentgenology, vol. 161, no. 6, pp. 1229-1235, 1993.

[4] R. Beck, "Image processing in the context of imaging science", in Image Processing, 1995. Proceedings., International Conference on, IEEE, vol. 2, 1995, pp. 304-307.

[5] A. Barrett, "Introduction to image processing", in Knowledge-Based Image Processing Systems, Springer, 1997, pp. 83-90.

[6] B. H. Brinkmann, A. Manduca, and R. A. Robb, "Optimized homomorphic unsharp masking for mr grayscale inhomogeneity correction", IEEE Transactions on Medical Imaging, vol. 17, no. 2, pp. 161-171, Apr. 1998, ISSN: 0278-0062. DOI: $10.1109 / 42.700729$.

[7] I. Pitas, Digital image processing algorithms and applications. John Wiley \& Sons, 2000.

[8] P.-S. Liao, T.-S. Chen, P.-C. Chung, et al., "A fast algorithm for multilevel thresholding", J. Inf. Sci. Eng., vol. 17, no. 5, pp. 713-727, 2001.

[9] S. A. Huettel, A. W. Song, G. McCarthy, et al., Functional magnetic resonance imaging. Sinauer Associates Sunderland, MA, 2004, vol. 1.

[10] S. K. Singh, C. Hawkins, I. D. Clarke, J. A. Squire, J. Bayani, T. Hide, R. M. Henkelman, M. D. Cusimano, and P. B. Dirks, "Identification of human brain tumour initiating cells", nature, vol. 432, no. 7015, p. 396, 2004.

[11] R. H. Baayen and R. Schreuder, "Morphological processing", Encyclopedia of Cognitive Science, 2006.

[12] S. V. Vaseghi, "Interpolation", in. May 2006, pp. 267-295. DOI: 10.1002/ 0470094966.ch10. 
[13] C. Calabrese, H. Poppleton, M. Kocak, T. L. Hogg, C. Fuller, B. Hamner, E. Y. Oh, M. W. Gaber, D. Finklestein, M. Allen, et al., "A perivascular niche for brain tumor stem cells", Cancer cell, vol. 11, no. 1, pp. 69-82, 2007.

[14] W. Y. Lo and S. M. Puchalski, "Digital image processing", Veterinary Radiology $\& 6$ Ultrasound, vol. 49, S42-S47, 2008.

[15] D.-Y. Huang and C.-H. Wang, "Optimal multi-level thresholding using a twostage otsu optimization approach", Pattern Recognition Letters, vol. 30, no. 3, pp. 275-284, 2009.

[16] A. K. Kaw and E. E. Kalu, Numerical methods with applications: (abridged edition). 2009.

[17] A. Kharrat, N. Benamrane, M. B. Messaoud, and M. Abid, "Detection of brain tumor in medical images", in 2009 3rd International Conference on Signals, Circuits and Systems (SCS), Nov. 2009, pp. 1-6. DOI: 10.1109/ICSCS.2009. 5412577.

[18] V. K. Mishra, S. Kumar, and N. Shukla, "Image acquisition and techniques to perform image acquisition", 2010.

[19] A. Yang, "Research on image filtering method to combine mathematics morphology with adaptive median filter", in 9th International Conference on Optical Communications and Networks (ICOCN 2010), Oct. 2010, pp. 55-59. DOI: 10.1049/cp.2010.1152.

[20] A. Krizhevsky, I. Sutskever, and G. E. Hinton, "Imagenet classification with deep convolutional neural networks", in Advances in neural information processing systems, 2012, pp. 1097-1105.

[21] A. Konieczka, J. Balcerek, and A. Dabrowski, "Iterative average filtering for image denoising", in 2013 Signal Processing: Algorithms, Architectures, Arrangements, and Applications (SPA), Sep. 2013, pp. 302-305.

[22] R. Yogamangalam and B. Karthikeyan, "Segmentation techniques comparison in image processing", International Journal of Engineering and Technology (IJET), vol. 5, no. 1, pp. 307-313, 2013.

[23] M. A. Elahi, A. Shahzad, M. Glavin, E. Jones, and M. O'Halloran, "Hybrid artifact removal for confocal microwave breast imaging", IEEE Antennas and Wireless Propagation Letters, vol. 13, pp. 149-152, 2014, ISSN: 1536-1225. DOI: 10.1109/LAWP.2014.2298975.

[24] B. R. Mohapatra, A. Mishra, and S. K. Rout, "A comprehensive review on image restoration techniques", International Journal of Research in Advent Technology, vol. 2, no. 3, pp. 101-105, 2014.

[25] P. S. Chahar and V. Thakare, "Performance comparison of various filters for removing gaussian and poisson noises", International Research Journal of Engineering and Technology, vol. 2, no. 5, pp. 1101-1105, 2015.

[26] K. N. Chaudhury, "Fast and accurate bilateral filtering using gauss-polynomial decomposition", in 2015 IEEE International Conference on Image Processing (ICIP), Sep. 2015, pp. 2005-2009. DOI: 10.1109/ICIP.2015.7351152. 
[27] R. Sanaei, K. Otto, K. Hölttä-Otto, and K. Wood, "Incorporating constraints in system modularization by interactive clustering of design structure matrices", Aug. 2016. DOI: 10.1115/DETC2016-60510.

[28] W. Mengqiao, Y. Jie, C. Yilei, and W. Hao, "The multimodal brain tumor image segmentation based on convolutional neural networks", in 2017 2nd IEEE International Conference on Computational Intelligence and Applications (ICCIA), Sep. 2017, pp. 336-339. DOI: 10.1109/CIAPP.2017.8167234.

[29] A. Min and Z. M. Kyu, "Mri images enhancement and tumor segmentation for brain", in Parallel and Distributed Computing, Applications and Technologies (PDCAT), 2017 18th International Conference on, IEEE, 2017, pp. 270-275.

[30] S. Sarwar, P. Panda, and K. Roy, "Gabor filter assisted energy efficient fast learning convolutional neural networks", May 2017.

[31] M. B. Shakir, M. A. Hossain, K. M. A. Shams, and F. R. Akib, "Early detection, segmentation and quantification of coronary artery blockage using efficient image processing technique", PhD thesis, BRAC University, 2017.

[32] R. Ezhilarasi and P. Varalakshmi, "Tumor detection in the brain using faster rcnn", in 2018 2nd International Conference on I-SMAC (IoT in Social, Mobile, Analytics and Cloud) (I-SMAC)I-SMAC (IoT in Social, Mobile, Analytics and Cloud) (I-SMAC), 2018 2nd International Conference on, Aug. 2018, pp. 388392. DOI: 10.1109/I-SMAC.2018.8653705.

[33] M. R. Islam and N. Rishad, "Effects of filter on the classification of brain mri image using convolutional neural network", in 2018 4th International Conference on Electrical Engineering and Information Communication Technology (iCEEiCT), Sep. 2018, pp. 489-494. DOI: 10.1109/CEEICT.2018.8628056.

[34] R. Vinoth and C. Venkatesh, "Segmentation and detection of tumor in mri images using cnn and svm classification", pp. 21-25, Mar. 2018. DOI: 10.1109/ ICEDSS.2018.8544306. 


\section{How to install $\mathrm{IAT}_{\mathrm{E}} \mathrm{X}$}

\section{Windows OS}

\section{TeXLive package - full version}

a) Download the TeXLive ISO (2.2GB) from https://www.tug.org/texlive/

b) Download WinCDEmu (if you don't have a virtual drive) from http://wincdemu.sysprogs.org/download/

c) To install Windows CD Emulator follow the instructions at http://wincdemu.sysprogs.org/tutorials/install/

d) Right click the iso and mount it using the WinCDEmu as shown in http://wincdemu.sysprogs.org/tutorials/mount/

e) Open your virtual drive and run setup.pl

or

\section{Basic MikTeX - $\mathrm{T}_{\mathrm{E}} \mathrm{X}$ distribution}

a) Download Basic-MiKTEX(32bit or 64bit) from http://miktex.org/download

b) Run the installer

c) To add a new package go to Start i¿ All Programs i¿ MikTex ¿i. Maintenance (Admin) and choose Package Manager

d) Select or search for packages to install

\section{TexStudio - $\mathrm{T}_{\mathrm{E}} \mathrm{X}$ editor}

a) Download TexStudio from http://texstudio.sourceforge.net/\#downloads

b) Run the installer 


\section{Mac OS X}

\section{MacTeX - $\mathrm{T}_{\mathrm{E}} \mathrm{X}$ distribution}

a) Download the file from https://www.tug.org/mactex/

b) Extract and double click to run the installer. It does the entire configuration, sit back and relax.

\section{TexStudio - $\mathrm{T}_{\mathrm{E}} \mathrm{X}$ editor}

a) Download TexStudio from

http://texstudio.sourceforge.net/\#downloads

b) Extract and Start

\section{Unix/Linux}

\section{TeXLive - $\mathrm{T}_{\mathrm{E}} \mathrm{X}$ distribution}

\section{Getting the distribution:}

a) TexLive can be downloaded from http://www.tug.org/texlive/acquire-netinstall.html.

b) TexLive is provided by most operating system you can use (rpm,apt-get or yum) to get TexLive distributions

\section{Installation}

a) Mount the ISO file in the mnt directory mount -t iso9660 -o ro,loop, noauto /your/texlive\#\#\#.iso /mnt

b) Install wget on your OS (use rpm, apt-get or yum install)

c) Run the installer script install-tl.

cd /your/download/directory ./install-tl

d) Enter command 'i' for installation

e) Post-Installation configuration: http://www.tug.org/texlive/doc/texlive-en/texlive-en.html\#x1-320003.4.1

f) Set the path for the directory of TexLive binaries in your .bashrc file 


\section{For 32bit OS}

For Bourne-compatible shells such as bash, and using Intel x86 GNU/Linux and a default directory setup as an example, the file to edit might be

edit $\$ \sim$.bashrc file and add following lines

PATH=/usr/local/texlive/2011/bin/i386-linux: \$PATH;

export PATH

MANPATH=/usr/local/texlive/2011/texmf/doc/man : \$MANPATH;

export MANPATH

INFOPATH=/usr/local/texlive/2011/texmf/doc/info: \$INFOPATH;

export INFOPATH

\section{For 64bit OS}

edit $\$ \sim$.bashrc file and add following lines

PATH=/usr/local/texlive/2011/bin/x86_64-linux: \$PATH;

export PATH

MANPATH=/usr/local/texlive/2011/texmf/doc/man : \$MANPATH;

export MANPATH

INFOPATH=/usr/local/texlive/2011/texmf/doc/info: \$INFOPATH;

export INFOPATH

\section{Fedora/RedHat/CentOS:}

sudo yum install texlive

sudo yum install psutils

\section{SUSE:}

sudo zypper install texlive

\section{Debian/Ubuntu:}

sudo apt-get install texlive texlive-latex-extra

sudo apt-get install psutils 


\section{Overleaf: GitHub for $\mathrm{IAT}_{\mathrm{E}} \mathrm{X}$ projects}

This Project was developed using Overleaf(https://www.overleaf.com/), an online LTEX editor that allows real-time collaboration and online compiling of projects to PDF format. In comparison to other $\mathrm{IT}_{\mathrm{E}} \mathrm{X}$ editors, Overleaf is a server-based application, which is accessed through a web browser. 